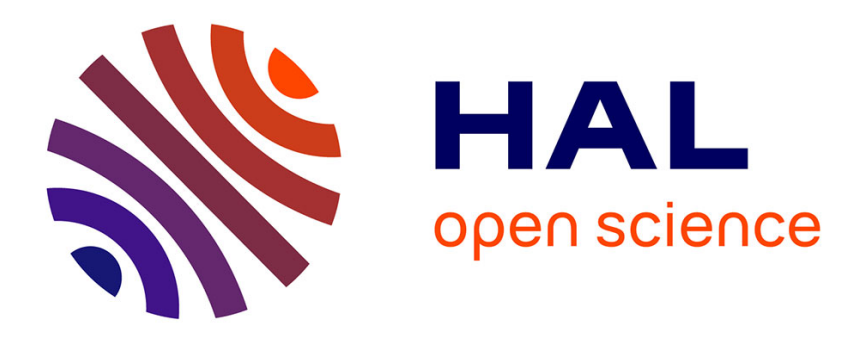

\title{
On a functional inequality arising in the analysis of finite-volume methods
}

Pierre Bousquet, Franck Boyer, Flore Nabet

\section{To cite this version:}

Pierre Bousquet, Franck Boyer, Flore Nabet. On a functional inequality arising in the analysis of finitevolume methods. Calcolo, 2016, 53 (3), pp.363-397. 10.1007/s10092-015-0153-0 . hal-01134988v3

\section{HAL Id: hal-01134988 \\ https://hal.science/hal-01134988v3}

Submitted on 26 Jun 2015

HAL is a multi-disciplinary open access archive for the deposit and dissemination of scientific research documents, whether they are published or not. The documents may come from teaching and research institutions in France or abroad, or from public or private research centers.
L'archive ouverte pluridisciplinaire HAL, est destinée au dépôt et à la diffusion de documents scientifiques de niveau recherche, publiés ou non, émanant des établissements d'enseignement et de recherche français ou étrangers, des laboratoires publics ou privés. 


\title{
ON A FUNCTIONAL INEQUALITY ARISING IN THE ANALYSIS OF FINITE-VOLUME METHODS
}

\author{
P BOUSQUET * F. BOYER $^{\dagger}$, AND F. NABET ${ }^{\dagger}$
}

\begin{abstract}
We establish a Poincaré-Wirtinger type inequality on some particular domains with a precise estimate of the constant depending only on the geometry of the domain. This type of inequality arises, for instance, in the analysis of finite volume (FV) numerical methods.

As an application of our result, we prove uniform a priori bounds for the FV approximate solutions of the heat equation with Ventcell boundary conditions in the natural energy space defined as the set of those functions in $H^{1}(\Omega)$ whose traces belong to $H^{1}(\partial \Omega)$. The main difficulty here comes from the fact that the approximation is performed on non-polygonal control volumes since the domain itself is non-polygonal.
\end{abstract}

Key words. Poincaré-Wirtinger inequality - Finite Volume methods - Ventcell boundary condition

AMS subject classifications. 35A23,35K05,65M08

1. Introduction. The main goal of this paper is to study functional inequalities of the following form

$$
\left|\frac{1}{m_{\sigma}} \int_{\sigma} u-\frac{1}{m_{\mathcal{K}}} \int_{\mathcal{K}} u\right| \leq C \operatorname{diam}(\mathcal{K}) \frac{1}{m_{\mathcal{K}}} \int_{\mathcal{K}}|D u|, \quad \forall u \in W^{1,1}\left(\mathbb{R}^{n}\right),
$$

where $\mathcal{K}$ is a bounded connected Lipschitz domain in $\mathbb{R}^{n}$ and $\sigma$ a non empty open subset of $\partial \mathcal{K}$. We have denoted by $m_{\mathcal{K}}$ the volume of $\mathcal{K}$ and $m_{\sigma}$ the surface measure of $\sigma$, namely its $(n-1)$-dimensional Hausdorff measure. Those notations will be used all along this paper.

The fact that such an inequality holds is straightforward, for instance by applying the Bramble-Hilbert lemma (see for instance [2]). Our main purpose is to estimate the dependence of the constant $C$ with respect to the geometry of the domain $\mathcal{K}$. In the particular case of a convex domain $\mathcal{K}$ the mean-value inequality immediately implies that

$$
\left|\frac{1}{m_{\sigma}} \int_{\sigma} u-\frac{1}{m_{\mathcal{K}}} \int_{\mathcal{K}} u\right| \leq \operatorname{diam}(\mathcal{K})\left(\sup _{\mathcal{K}}|D u|\right), \forall u \in W^{1, \infty}\left(\mathbb{R}^{n}\right),
$$

with no geometric constant in the right-hand side. The inequality (1.1) has to be seen as a generalisation of (1.2) to less regular functions $u$. This loss of regularity induces that the constant in the inequality may depend on the shape of $\mathcal{K}$. Observe that, if $\mathcal{K}$ is not convex, one has to replace $\sup _{\mathcal{K}}|D u|$ by $\sup _{\operatorname{Conv}(\mathcal{K})}|D u|$ in $(1.2)$, where $\operatorname{Conv}(\mathcal{K})$ is the convex hull of $\mathcal{K}$.

Inequalities of the form (1.1) play an important role in the analysis of finite volume numerical methods for elliptic or parabolic equations on general meshes, which is our main motivation. They are meant to be applied to each cell (control volume) $\mathcal{K}$ in a mesh of a given computational domain. They allow to prove stability estimates in (discrete) Sobolev spaces for the natural $L^{2}$ projections of the functions defined on $\Omega$ and the projections of their traces. To our knowledge, such inequalities have only been established up to now in the framework of polygonal sets $\mathcal{K}$. However, for more complex situations, like for the discretisation of the heat equation with dynamic Ventcell boundary conditions, we are interested in proving such inequalities for non polygonal domains. We detail such an application in Section 6

* pierre.bousquet@math.univ-toulouse.fr. Institut de Mathématiques de Toulouse, UMR 5219, Université de Toulouse, F-31062 Toulouse Cedex 9, France

$\dagger\{$ franck.boyer, flore.nabet\} @univ-amu.fr. Aix Marseille Université, CNRS, Centrale Marseille, I2M, UMR 7373, 13453 Marseille, France 
Let us mention some references where such inequalities are proved and/or used in the finite volume framework.

- In [7, Lemma 3.4] (see also [4, Lemma 7.2] and [5, Lemmas 6.2 and 6.3]), (1.1) is proved (in $2 \mathrm{D}$ for simplicity) when $\mathcal{K}$ is polygonal and convex, with a constant $C$ depending only on the number of edges/faces of $\mathcal{K}$, and on the shape-regularity ratio $(\operatorname{diam}(\mathcal{K}))^{2} / m_{\mathcal{K}}$.

- In [6. Lemma 6.6], the inequality is slightly generalized to a polygonal $\mathcal{K}$ which is simply supposed to be star-shaped with respect to a suitable ball.

- In [1], such inequalities are used for the convergence and error analysis of some approximation of non-linear Leray-Lions type operators (a model of which is the $p$-Laplace problem).

Finally, we refer to [9] for an example of analysis of a more complex model of a non-linear evolution equation associated with a non-linear dynamical boundary condition. This reference was in fact our main motivation for the present work. Indeed, compared to the other references above, the numerical method in [9] is derived on non-polygonal control volumes, so that an inequality like $(1.1)$ is needed on non-polygonal open sets $\mathcal{K}$, see also Section 6

The outline of the paper is the following. In Section 2, we state our main result (Theorem 2.1 in 2D) and the geometric assumptions that we shall work with in the sequel. Section 3 is devoted to the proof of the main result whereas in Section 4, we state and prove a sort of Poincaré-Wirtinger inequality related to the functional inequality proved in Theorem 2.1. Section 5 is dedicated to the extension of our main inequality in the higher dimensional case (i.e. in $\mathbb{R}^{n}$, for $n \geq 3$ ). Finally, in order to illustrate this work, we provide an application, as simple as possible, of Theorem 2.1 to the proof of uniform discrete energy estimates for a finite volume approximation of a toy system on a non-polygonal domain $\Omega$.

2. Main result. Given a $C^{1}$ curve $\sigma \subset \mathbb{R}^{2}$ and a point $z_{*} \in \mathbb{R}^{2} \backslash \sigma$, we consider the following domain $T$ :

$$
T=\left\{z_{*}+t\left(\gamma(\theta)-z_{*}\right): t \in\right] 0,1[, \theta \in] 0,1[\},
$$

where $\gamma:[0,1] \rightarrow \mathbb{R}^{2}$ is a $C^{1}$ parametrization of $\sigma: \gamma([0,1])=\sigma, \gamma$ is one-to-one and $\left|\gamma^{\prime}\right|$ does not vanish. Without loss of generality, we choose the parametrization $\gamma$ in such a way that $\left|\gamma^{\prime}(\theta)\right|=m_{\sigma}$ for every $\theta \in[0,1]$.

We say that $T$ is a pseudo-triangle if for every $x \in \sigma$,

$$
\left\{z_{*}+t\left(x-z_{*}\right): t \geq 0\right\} \cap \sigma=\{x\} .
$$
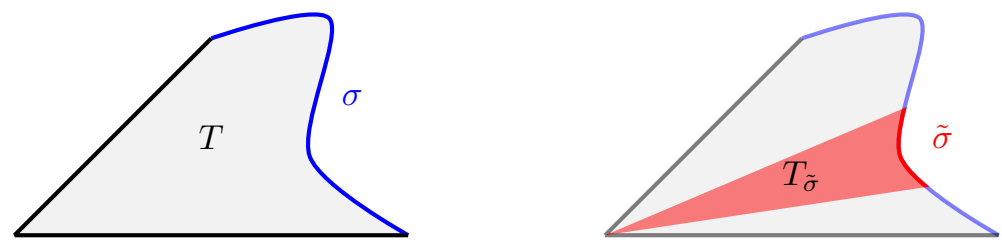

FIG. 2.1. The pseudo-triangle $T$ with its curved edge $\sigma$ and one of its sub-triangle

Without loss of generality, we shall assume that the vertex $z_{*}$ of $T$ opposite to $\sigma$ is the origin $(0,0)$ of $\mathbb{R}^{2}$.

THEOREM 2.1. Let $T$ be a pseudo-triangle as above. We assume that there exist $\mu, \nu>0$ such that for any sub-arc $\tilde{\sigma} \subset \sigma$, the corresponding sub-triangle $T_{\tilde{\sigma}}$ (see Figure 2.1 ) satisfies 


$$
\mu \leq \frac{m_{T_{\tilde{\sigma}}}}{m_{\tilde{\sigma}}} \leq \nu
$$

Then for every $p \in\left[1,+\infty\left[\right.\right.$ and every $u \in W^{1, p}(T)$,

$$
\left|\frac{1}{m_{\sigma}} \int_{\sigma} u-\frac{1}{m_{T}} \int_{T} u\right|^{p} \leq C^{p}\left(m_{\sigma}+\operatorname{diam}(T)\right)^{p} \frac{1}{m_{T}} \int_{T}|D u|^{p},
$$

where $C$ only depends on the ratio $\frac{\nu}{\mu}$.

As already noticed in the introduction, the main issue is to understand how the constant in the inequality depends on the geometry of this pseudo-triangle $T$.

REMARK 2.1. For a real flat triangle $T$, the quantity $\frac{m_{T_{\tilde{\tau}}}}{m_{\tilde{\sigma}}}$ does not depend on $\tilde{\sigma}$ and is equal to $\frac{m_{T}}{m_{\sigma}}$. In this particular case, we have $\mu=\nu$ and $m_{\sigma} \leq \operatorname{diam}(T)$. Hence, we recover exactly the inequality proved in [7].

PROPOSITION 2.2. Under assumption 2.1), the map $\theta \mapsto \operatorname{det}\left(\gamma(\theta), \gamma^{\prime}(\theta)\right)$ is either nonnegative everywhere or nonpositive everywhere.

In the sequel, we assume that the orientation is chosen such that $\operatorname{det}\left(\gamma, \gamma^{\prime}\right) \geq 0$. Then, assumption (2.2) is equivalent to the following inequality

$$
2 \mu m_{\sigma} \leq \operatorname{det}\left(\gamma(\theta), \gamma^{\prime}(\theta)\right) \leq 2 \nu m_{\sigma}, \quad \forall \theta \in[0,1] .
$$

Proof. One can assume without loss of generality that $\gamma([0,1])$ is contained in the half plane $\left\{(x, y) \in \mathbb{R}^{2}: x>0\right\}$. There exists a $\left.C^{1} \operatorname{map} \varphi:[0,1] \rightarrow\right]-\pi / 2, \pi / 2[$ such that for every $\theta \in[0,1]$,

$$
\frac{\gamma(\theta)}{|\gamma(\theta)|}=(\cos \varphi(\theta), \sin \varphi(\theta))
$$

By (2.1), the map $\varphi$ is one-to-one. Hence, it is either strictly increasing or strictly decreasing. Assume for instance that $\varphi$ is strictly increasing.

This implies that for every $\theta \in[0,1)$, for every $h>0$ such that $\theta+h \in[0,1]$,

$$
\begin{aligned}
\operatorname{det}\left(\gamma(\theta), \frac{\gamma(\theta+h)-\gamma(\theta)}{h}\right) & =\frac{1}{h} \operatorname{det}(\gamma(\theta), \gamma(\theta+h)) \\
& =\frac{1}{h}|\gamma(\theta)||\gamma(\theta+h)| \sin (\varphi(\theta+h)-\varphi(\theta)) \geq 0 .
\end{aligned}
$$

Passing to the limit $h \rightarrow 0$ yields the desired result. Then

Assume now that (2.2) holds true, then let $0 \leq a<b \leq 1$, and consider $\tilde{\sigma}=\gamma([a, b])$.

$$
m_{\tilde{\sigma}}=(b-a) m_{\sigma} \text { and } m_{T_{\tilde{\sigma}}}=\frac{1}{2} \int_{a}^{b} \operatorname{det}\left(\gamma(\theta), \gamma^{\prime}(\theta)\right) d \theta,
$$

thus thanks to 2.2,

$$
\mu \leq \frac{\int_{a}^{b} \operatorname{det}\left(\gamma(\theta), \gamma^{\prime}(\theta)\right) d \theta}{2(b-a) m_{\sigma}} \leq \nu
$$


and we obtain 2.4 when $b$ tends to $a$. Conversely, assume that 2.4 holds. Then 2.2 follows by integration of (2.4) on the segment $[a, b]$.

REMARK 2.2. For some particular cases, we can estimate the constant in the inequality (2.3) even if the pseudo-triangle $T$ does not satisfy assumption (2.2). In order to illustrate such a situation, we consider the pseudo-triangle $T$ defined as follows:

$$
T=\left\{t \gamma(\theta): 0<t<1,-\theta_{0}<\theta<\pi+\theta_{0}\right\} \text {, with } \gamma(\theta)=\left(R \cos \theta, R \sin \theta+\frac{R}{\sin \left(\theta_{0}\right)}\right),
$$

where $0<\theta_{0}<\frac{\pi}{2}, R>0$. Observe that $\gamma$ and $\gamma^{\prime}$ are colinear for $\theta=-\theta_{0}$ or $\theta=\pi+\theta_{0}$ so that assumption (2.2) is not satisfied here. We decompose the pseudo-triangle $T$ into the piece of disk $P$ of radius $R$ and center $A=\left(0, R / \sin \left(\theta_{0}\right)\right)$ and the quadrilateral $Q$ defined by: $Q=T \backslash P$.

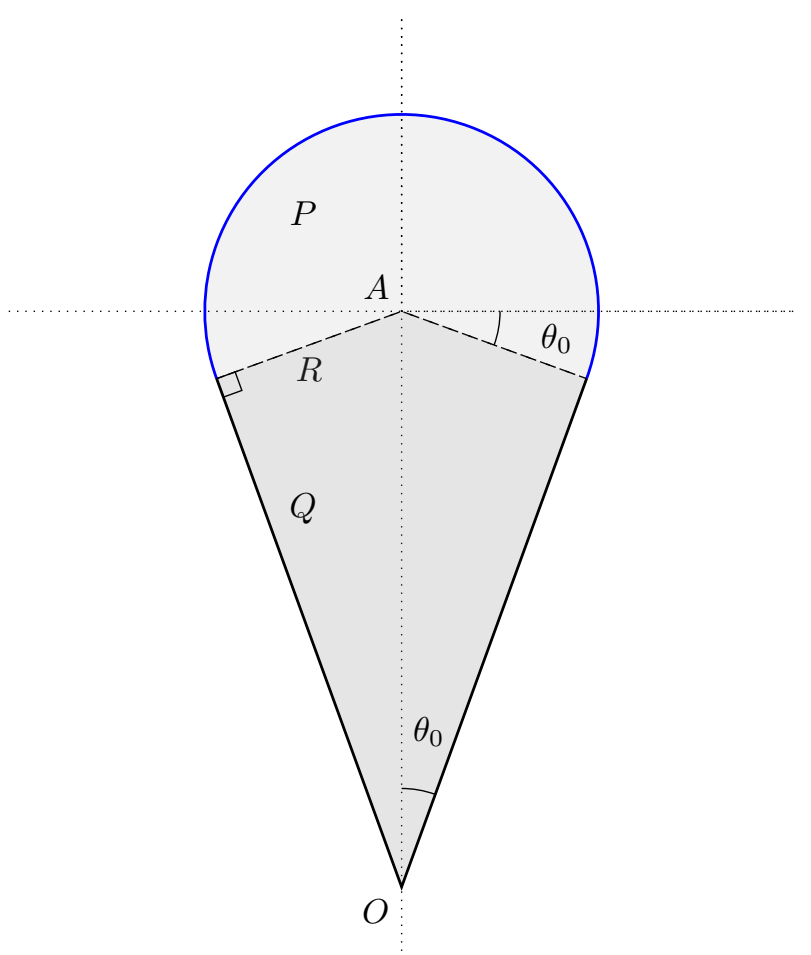

FIG. 2.2. A particular case which does not satisfy assumption 2.2

First, we remark that assumption (2.2) is satisfied for the pseudo-triangle $P$ with the ratio $\frac{\nu}{\mu}$ equal to 1 (see Remark 2.1). Then we can apply Theorem 2.1 to the pseudo-triangle $P$, so that there exists a constant $C_{0}>0$ which does not depend on $R$ and $\theta_{0}$ such that

$$
\left|\frac{1}{m_{\sigma}} \int_{\sigma} u-\frac{1}{m_{P}} \int_{P} u\right| \leq C_{0} R \frac{1}{m_{P}} \int_{P}|D u| .
$$

Moreover, since $P \subset T$ and $T$ is convex, we can apply [4] Lemma 7.1],

$$
\left|\frac{1}{m_{T}} \int_{T} u-\frac{1}{m_{P}} \int_{P} u\right| \leq \widetilde{C_{0}} \frac{(\operatorname{diam}(T))^{3}}{m_{P} m_{T}} \int_{T}|D u|
$$


Now, we want to control the volume of the pseudo-triangle $T$ by the volume of $P$. We note that

$$
m_{Q}=\frac{R^{2}}{\tan \theta_{0}} \quad, \quad m_{P}=\frac{R^{2}}{2}\left(\pi+2 \theta_{0}\right) \quad, \quad \operatorname{diam}(T)=R\left(1+\frac{1}{\sin \theta_{0}}\right) .
$$

Hence

$$
m_{Q} \leq \frac{\operatorname{diam}(T)}{R} \frac{2}{\pi} m_{P}
$$

and then using that $R \leq \operatorname{diam}(T)$,

$$
m_{P} \geq \frac{\pi R}{\pi R+2 \operatorname{diam}(T)} m_{T} \geq C_{1} \frac{R}{\operatorname{diam}(T)} m_{T}
$$

This implies

$$
\left|\frac{1}{m_{\sigma}} \int_{\sigma} u-\frac{1}{m_{P}} \int_{P} u\right| \leq \frac{C_{0}}{C_{1}} \operatorname{diam}(T) \frac{1}{m_{T}} \int_{T}|D u|,
$$

and

$$
\left|\frac{1}{m_{T}} \int_{T} u-\frac{1}{m_{P}} \int_{P} u\right| \leq \frac{\widetilde{C_{0}}}{C_{1}} \frac{(\operatorname{diam}(T))^{4}}{R m_{T}^{2}} \int_{T}|D u| .
$$

Since $R \leq \operatorname{diam}(T), m_{T} \leq(\operatorname{diam}(T))^{2}$, the above two inequalities yield

$$
\begin{aligned}
\left|\frac{1}{m_{T}} \int_{T} u-\frac{1}{m_{\sigma}} \int_{\sigma} u\right| & \leq C \frac{(\operatorname{diam}(T))^{4}}{R m_{T}^{2}} \int_{T}|D u| \\
& \leq \frac{C^{\prime}}{\sin \left(\theta_{0}\right)^{2}} \operatorname{diam}(T) \frac{1}{m_{T}} \int_{T}|D u|,
\end{aligned}
$$

where $C^{\prime}$ is a universal constant. As expected we observe that the above inequality does not depend on $R$ and blows up when $\theta_{0}$ goes to 0 .

3. Proof of Theorem 2.1. By Jensen's inequality, we only need to establish the case $p=1$. We begin by proving a change of variables formula (Proposition 3.1p that let us express the differences between the mean values of a function on $T$ and on $\sigma$ as a weighted integral of its gradient on $T$. Then, we prove in Proposition 3.2 that this change of variables can be realized with a diffeomorphism satisfying suitable estimates. This proposition relies on two technical Lemmas 3.3 and 3.4. Theorem 2.1 readily follows from those two propositions.

We notice that the existence of such a diffeomorphism is ensured by a general result of [3] but we have to be able to estimate the derivatives of this diffeomorphism in function of the geometry of $T$. That is why we resume explicitly the steps of [3] that allow us to control all the constants involved in the estimates.

In the sequel, we denote by $\left.Q^{2}=\right] 0,1\left[{ }^{2}\right.$ the unit cube in $\mathbb{R}^{2}$. By a standard approximation argument, one can assume that $\gamma \in C^{2}(0,1)$.

PROPOSITION 3.1. Assume that there exists a Lipschitz continuous map $\Phi: \overline{Q^{2}} \rightarrow \bar{T}$ such that

1. $\Phi$ is a $C^{1}$ diffeomorphism from $Q^{2}$ onto $T$,

2. $\Phi(0, \theta)=(0,0)$,

3. $\Phi(1, \theta)=\gamma(\theta)$, 
4. $\operatorname{Jac} \Phi(s, \theta)=2 m_{T} s$.

Then for every $u \in W^{1,1}(T)$, we have

$$
\frac{1}{m_{\sigma}} \int_{\sigma} u-\frac{1}{m_{T}} \int_{T} u=\frac{1}{2 m_{T}} \int_{T} D u(x, y)\left[s \partial_{s} \Phi(s, \theta)\right]_{(s, \theta)=\Phi^{-1}(x, y)} d x d y .
$$

Proof. It follows from (2.4) that the pseudo-triangle $T$ is biLipschitz homeomorphic to a (true) triangle, see the proof of Lemma 4.2 in section 4. In particular, $T$ is a Lipschitz domain. By a standard density argument, we can thus assume that $u \in C^{1}(\bar{T})$. Let

$$
v(s, \theta)=u \circ \Phi(s, \theta) \mathbf{J a c} \Phi(s, \theta) .
$$

Then for every $(t, \theta) \in Q^{2}$,

$$
v(1, \theta)-v(t, \theta)=\int_{t}^{1} \partial_{s} v(s, \theta) d s .
$$

Hence, thanks to the assumptions on $\Phi$, we obtain

$$
2 m_{T} u(\gamma(\theta))-u \circ \Phi(t, \theta) \operatorname{Jac} \Phi(t, \theta)=\int_{t}^{1}\left(\partial_{s}(u \circ \Phi) \mathbf{J a c} \Phi+2 m_{T}(u \circ \Phi)\right) d s .
$$

By integrating this equality on $Q^{2}$ and using an obvious change of variables, we get

$$
2 \frac{m_{T}}{m_{\sigma}} \int_{\sigma} u-\int_{T} u=\int_{0}^{1} d \theta \int_{0}^{1} d t \int_{t}^{1}\left(\partial_{s}(u \circ \Phi) \mathrm{Jac} \Phi+2 m_{T}(u \circ \Phi)\right) d s .
$$

By Fubini theorem,

$$
\begin{aligned}
\int_{0}^{1} d \theta \int_{0}^{1} d t \int_{t}^{1}\left(\partial_{s}(u \circ \Phi) \operatorname{Jac} \Phi+\right. & \left.2 m_{T}(u \circ \Phi)\right) d s \\
& =\int_{Q^{2}} s\left(\partial_{s}(u \circ \Phi) \operatorname{Jac} \Phi+2 m_{T}(u \circ \Phi)\right) d s d \theta .
\end{aligned}
$$

Since $\operatorname{Jac} \Phi(s, \theta)=2 m_{T} s$, the same change of variables gives

$$
\begin{aligned}
\int_{Q^{2}} s\left(\partial_{s}(u \circ \Phi) \mathbf{J a c} \Phi+2 m_{T}\right. & (u \circ \Phi)) d s d \theta \\
& =\int_{T} D u(x, y)\left[s \partial_{s} \Phi(s, \theta)\right]_{(s, \theta)=\Phi^{-1}(x, y)} d x d y+\int_{T} u .
\end{aligned}
$$

The claim now follows from this identity together with 3.1 .

The proof of Theorem 2.1, that is the one of the inequality [2.3), is now a straightforward consequence of the following proposition that claims that we can build a suitable $\Phi$ and apply Proposition 3.1

PROPOSITION 3.2. Under assumption 2.2, there exists a universal constant $C>0$ and a map $\Phi: \overline{Q^{2}} \rightarrow \bar{T}$ satisfying the properties required in Proposition 3.1 and the additional estimate

$$
\left|\partial_{s} \Phi(s, \theta)\right| \leq C \frac{\nu^{3}}{\mu^{3}}\left(\operatorname{diam}(T)+m_{\sigma}\right), \quad \forall(s, \theta) \in Q^{2} .
$$


The sequel of this section is thus devoted to the proof of Proposition 3.2 We first observe that, in the case when $T$ is a real triangle, namely when $\sigma$ is a segment, the map $\Phi=\phi_{2}$ with

$$
\phi_{2}:(s, \theta) \in \overline{Q^{2}} \mapsto s \gamma(\theta) \in \bar{T},
$$

satisfies the assumptions of Proposition 3.1 as well as estimate 3.2 .

In the general case where $\sigma$ is curved, we have

$$
\operatorname{Jac} \phi_{2}(s, \theta)=s \operatorname{det}\left(\gamma(\theta), \gamma^{\prime}(\theta)\right),
$$

and this quantity depends on $\theta$ : we cannot choose $\Phi=\phi_{2}$ anymore. Thus, we are going to right compose $\phi_{2}$ with a diffeomorphism of the unit cube to construct a $\Phi: \overline{Q^{2}} \rightarrow \bar{T}$ satisfying Proposition 3.2, see Figure 3.1 .

To simplify the notation, we define $g$ by

$$
g:(s, \theta) \in \overline{Q^{2}} \mapsto \operatorname{Jac} \phi_{2}(s, \theta)=s \operatorname{det}\left(\gamma, \gamma^{\prime}\right)(\theta) .
$$

Then, we construct a first diffeomorphism $\phi_{1}$ of $Q^{2}$ such that $\phi_{2} \circ \phi_{1}$ satisfies the first three assumptions of Proposition 3.1 as a well as a weaker version (integrated with respect to $s$ ) of the third assumption. Obtaining the strong version of this property (that is a point-by-point equality) will be the purpose of Lemma 3.4

LEMma 3.3. There exists a $C^{1}$ diffeomorphism $\phi_{1}: \overline{Q^{2}} \rightarrow \overline{Q^{2}}$ such that

1. for every $x \in \partial Q^{2}, \phi_{1}(x)=x$,

2. for every $\theta \in[0,1]$,

$$
\int_{0}^{1} \operatorname{Jac}\left(\phi_{2} \circ \phi_{1}\right)(s, \theta) d s=\int_{0}^{1} g \circ \phi_{1}(s, \theta) \operatorname{Jac} \phi_{1}(s, \theta) d s=m_{T} .
$$

3. for every $(s, \theta) \in \overline{Q^{2}}$,

$$
\frac{\mu}{4 \nu} \leq \operatorname{Jac} \phi_{1}(s, \theta) \leq \frac{5 \nu}{\mu}
$$

Proof. Let

$$
\varepsilon=\frac{\mu}{10 \nu}
$$

and $\zeta \in C_{c}^{\infty}(0,1)$ be a cut-off function such that $0 \leq \zeta \leq 1+\varepsilon,\left|\zeta^{\prime}\right|_{L^{\infty}} \leq \frac{10}{\varepsilon}$ and

$$
\int_{0}^{1} \zeta(s) d s=1 \text { and } \int_{0}^{1}|\zeta(s)-1| d s<\varepsilon .
$$

We introduce the map

$$
G:(a, b) \mapsto \int_{0}^{1} s d s \int_{0}^{a+\zeta(s) b} \operatorname{det}\left(\gamma, \gamma^{\prime}\right)(\theta) d \theta .
$$

Then $G$ is well-defined and $C^{2}$ on the set $\left\{(a, b): 0 \leq a \leq 1, \frac{-a}{1+\varepsilon} \leq b \leq \frac{1-a}{1+\varepsilon}\right\}$ (here, we use the fact that $\gamma \in C^{2}([0,1])$ so that $\operatorname{det}\left(\gamma, \gamma^{\prime}\right)$ is $\left.C^{1}([0,1])\right)$. Moreover, we have

$$
\partial_{a} G(a, b)=\int_{0}^{1} \operatorname{det}\left(\gamma, \gamma^{\prime}\right)(a+\zeta(s) b) s d s
$$




$$
\partial_{b} G(a, b)=\int_{0}^{1} \operatorname{det}\left(\gamma, \gamma^{\prime}\right)(a+\zeta(s) b) \zeta(s) s d s
$$

By (2.4), we have

$$
2 m_{\sigma} \mu \int_{0}^{1} \zeta(s) s d s \leq \partial_{b} G(a, b) \leq 2 m_{\sigma} \nu \int_{0}^{1} \zeta(s) s d s .
$$

We can bound the right hand side by $2 m_{\sigma} \nu$ while

$$
\begin{aligned}
2 m_{\sigma} \mu \int_{0}^{1} \zeta(s) s d s & \geq 2 m_{\sigma} \mu\left(\int_{0}^{1} s d s-\int_{0}^{1}|\zeta(s)-1| d s\right) \\
& \geq 2 m_{\sigma} \mu\left(\int_{0}^{1} s d s-\varepsilon\right) \geq \frac{4 m_{\sigma} \mu}{5}
\end{aligned}
$$

Hence we conclude that

$$
0<\frac{4 m_{\sigma} \mu}{5} \leq \partial_{b} G(a, b) \leq 2 m_{\sigma} \nu
$$

We claim that

$$
G\left(a, \frac{-a}{1+\varepsilon}\right) \leq m_{T} a
$$

Indeed, by 2.4,

$$
G\left(a, \frac{-a}{1+\varepsilon}\right) \leq 2 m_{\sigma} \nu \int_{0}^{1} s\left(a-\frac{a}{1+\varepsilon} \zeta(s)\right) d s \leq \frac{2 m_{\sigma} \nu a}{1+\varepsilon} \int_{0}^{1}(1+\varepsilon-\zeta(s)) d s .
$$

By (3.6), this implies

$$
G\left(a, \frac{-a}{1+\varepsilon}\right) \leq \frac{2 m_{\sigma} \nu a \varepsilon}{1+\varepsilon} \leq 2 m_{\sigma} \nu a \varepsilon
$$

Since

$$
m_{T}=\frac{1}{2} \int_{0}^{1} \operatorname{det}\left(\gamma, \gamma^{\prime}\right)(\theta) d \theta \geq m_{\sigma} \mu
$$

it follows from 3.5 that

$$
G\left(a, \frac{-a}{1+\varepsilon}\right) \leq m_{T} a
$$

which proves our claim.

Similarly, one can prove that

$$
\int_{0}^{1} s d s \int_{a+\zeta(s) \frac{1-a}{1+\varepsilon}}^{1} \operatorname{det}\left(\gamma, \gamma^{\prime}\right)(\theta) d \theta \leq m_{T}(1-a)
$$

This can be written as

$$
G(1,0)-G\left(a, \frac{1-a}{1+\varepsilon}\right) \leq m_{T}(1-a) .
$$


Since

$$
G(1,0)=\int_{0}^{1} s d s \int_{0}^{1} \operatorname{det}\left(\gamma, \gamma^{\prime}\right)(\theta) d \theta=m_{T}
$$

this implies

$$
G\left(a, \frac{1-a}{1+\varepsilon}\right) \geq m_{T} a
$$

We deduce from (3.7), (3.8) and (3.9) that for every $a \in[0,1]$, there exists a unique $w(a) \in$ $[-a /(1+\varepsilon),(1-a) /(1+\varepsilon)]$ such that

$$
G(a, w(a))=m_{T} a .
$$

By the implicit function theorem, the function $w$ is $C^{2}$ on $[0,1]$ and satisfies

$$
\partial_{a} G(a, w(a))+\partial_{b} G(a, w(a)) w^{\prime}(a)=m_{T} .
$$

Since $G(0,0)=0$ and $G(1,0)=m_{T}$, we have $w(0)=0=w(1)$.

We claim that for every $s \in[0,1]$, for every $a \in[0,1]$,

$$
1+\zeta(s) w^{\prime}(a)>0
$$

To this end, it would be sufficient to prove that $\partial_{b} G(a, w(a))\left(1+\zeta(s) w^{\prime}(a)\right)>0$ but for a further use we shall derive more precise bounds on this quantity.

- By 3.10, we can write

(3.11) $\partial_{b} G(a, w(a))\left(1+\zeta(s) w^{\prime}(a)\right)=\partial_{b} G(a, w(a))+\zeta(s)\left(m_{T}-\partial_{a} G(a, w(a))\right)$,

and thus

$$
\begin{aligned}
\partial_{b} G(a, w(a))\left(1+\zeta(s) w^{\prime}(a)\right)= & \partial_{b} G(a, w(a))-\partial_{a} G(a, w(a)) \\
& +\zeta(s) m_{T}+(1-\zeta(s)) \partial_{a} G(a, w(a)) .
\end{aligned}
$$

But

$$
\begin{aligned}
\partial_{b} G(a, w(a))-\partial_{a} G(a, w(a)) & =\int_{0}^{1} \operatorname{det}\left(\gamma, \gamma^{\prime}\right)(a+\zeta(s) w(a))(\zeta(s)-1) s d s \\
& \geq-2 \nu m_{\sigma} \int_{0}^{1}|\zeta-1| \geq-2 \nu m_{\sigma} \varepsilon=-\frac{\mu m_{\sigma}}{5} .
\end{aligned}
$$

In the last inequality, we have used 3.6. Moreover, since $0 \leq \zeta \leq 1+\varepsilon$,

$$
\begin{aligned}
\zeta(s) m_{T}+(1-\zeta(s)) \partial_{a} G(a, w(a)) & \\
& \geq \min \left(\partial_{a} G(a, w(a)),(1+\varepsilon) m_{T}-\varepsilon \partial_{a} G(a, w(a))\right) .
\end{aligned}
$$

Since $m_{\sigma} \mu \leq \partial_{a} G(a, w(a)) \leq m_{\sigma} \nu$ and $m_{T} \geq m_{\sigma} \mu$, we get by 3.5

$$
\zeta(s) m_{T}+(1-\zeta(s)) \partial_{a} G(a, w(a)) \geq \frac{9 m_{\sigma} \mu}{10} .
$$

This implies

$$
\partial_{b} G(a, w(a))\left(1+\zeta(s) w^{\prime}(a)\right) \geq \frac{m_{\sigma} \mu}{2} .
$$


- Using that $m_{T} \leq m_{\sigma} \nu$ and that $\partial_{a} G>0$, we obtain by (3.7) and (3.11)

$$
\begin{aligned}
\partial_{b} G(a, w(a))\left(1+\zeta(s) w^{\prime}(a)\right) & \leq \partial_{b} G(a, w(a))+\zeta(s) m_{T} \\
& \leq 2 \nu m_{\sigma}+(1+\varepsilon) m_{\sigma} \nu \leq 4 m_{\sigma} \nu .
\end{aligned}
$$

Gathering 3.7), 3.12) and 3.13, we deduce that

$$
\frac{\mu}{4 \nu} \leq 1+\zeta(s) w^{\prime}(a) \leq 5 \frac{\nu}{\mu} .
$$

We now define

$$
\phi_{1}(s, \theta)=(s, \theta+\zeta(s) w(\theta)) \quad, \quad(s, \theta) \in \overline{Q^{2}} .
$$

It appears that $\phi_{1}$ is $C^{1}$ on $Q^{2}$ and satisfies $\operatorname{Jac} \phi_{1}(s, \theta)=1+\zeta(s) w^{\prime}(\theta)>0$. Since for every $s \in[0,1]$, the function $\theta \mapsto \theta+\zeta(s) w(\theta)$ is continuous and increasing, it maps $[0,1]$ onto $[0,1]$. Hence $\phi_{1}$ is a $C^{1}$ diffeomorphism from $\overline{Q^{2}}$ onto $\overline{Q^{2}}$. Moreover, $\phi_{1}$ agrees with the identity map on $\partial Q^{2}$. We now turn to the proof of (3.3).

Let $a \in[0,1]$. By definition of $w$ and $g$,

$$
\int_{0}^{1} d s \int_{0}^{a+\zeta(s) w(a)} g(s, \theta) d \theta=m_{T} a .
$$

By definition of $\phi_{1}$, this can be written

$$
\int_{\phi_{1}((0,1) \times(0, a))} g=m_{T} a .
$$

By the change of variables formula, this gives

$$
\int_{0}^{a} d \theta \int_{0}^{1} g \circ \phi_{1}(s, \theta) \mathbf{J a c} \phi_{1}(s, \theta) d s=m_{T} a .
$$

Since this holds true for any $a \in[0,1]$, by derivation we deduce

$$
\int_{0}^{1} g \circ \phi_{1}(s, \theta) \mathbf{J a c} \phi_{1}(s, \theta) d s=m_{T}, \quad \forall \theta \in(0,1) ;
$$

which completes the proof of 3.3 .

Since Jac $\phi_{1}(s, \theta)=1+\zeta(s) w^{\prime}(\theta)$, we can use (3.14), to obtain the estimate (3.4). The lemma is proven.

We proceed with the construction of the diffeomorphism $\Phi$ that we search in the form $\phi_{2} \circ \phi_{1} \circ \phi_{0}$. To simplify the notation, we consider the map $g_{1}: Q^{2} \rightarrow \mathbb{R}$ defined as follows:

$$
\begin{aligned}
g_{1}(s, \theta) & =\operatorname{Jac}\left(\phi_{2} \circ \phi_{1}\right)(s, \theta) \\
& =g \circ \phi_{1}(s, \theta) \operatorname{Jac} \phi_{1}(s, \theta) \\
& =s \operatorname{det}\left(\gamma, \gamma^{\prime}\right)(\theta+\zeta(s) w(\theta))\left(1+\zeta(s) w^{\prime}(\theta)\right) .
\end{aligned}
$$

Observe that for every $s \in(0,1], \theta \in[0,1], g_{1}(s, \theta)>0$.

LEMMA 3.4. There exists a Lipschitz homeomorphism $\phi_{0}: \overline{Q^{2}} \rightarrow \overline{Q^{2}}$ which is $C^{1}$ on $Q^{2}$ and such that

1. for every $\theta \in[0,1], \phi_{0}(0, \theta)=(0, \theta)$ and $\phi_{0}(1, \theta)=(1, \theta)$, 
2. for every $(s, \theta) \in Q^{2}$,

$$
\operatorname{Jac}\left(\phi_{2} \circ \phi_{1} \circ \phi_{0}\right)(s, \theta)=g_{1} \circ \phi_{0}(s, \theta) \operatorname{Jac} \phi_{0}(s, \theta)=2 m_{T} s .
$$

3. for every $(s, \theta) \in Q^{2}$,

$$
\left|\partial_{s} \phi_{0}(s, \theta)\right| \leq C
$$

where $C$ only depends on $\nu / \mu$.

Proof. For every $(s, \theta) \in \overline{Q^{2}}$, we denote by $v(s, \theta)$ the unique element of $[0,1]$ such that

$$
\int_{0}^{v(s, \theta)} g_{1}\left(s^{\prime}, \theta\right) d s^{\prime}=m_{T} s^{2} .
$$

The map $v$ is well-defined since $g_{1}(s, \theta)>0$ for every $s \in(0,1], \theta \in[0,1]$ and also because

$$
\int_{0}^{1} g_{1}\left(s^{\prime}, \theta\right) d s^{\prime}=m_{T}
$$

This is exactly the reason why we constructed $\phi_{1}$ in Lemma 3.3 Moreover, $v(0, \theta)=0$ and $v(1, \theta)=1$. By the implicit function theorem, $v$ is $C^{1}$ on $(0,1] \times[0,1]$ and satisfies $g_{1}(v(s, \theta), \theta) \partial_{s} v(s, \theta)=2 m_{T} s$; that is,

$$
v(s, \theta) \operatorname{det}\left(\gamma, \gamma^{\prime}\right)(\theta+\zeta \circ v(s, \theta) w(\theta))\left(1+\zeta \circ v(s, \theta) w^{\prime}(\theta)\right) \partial_{s} v(s, \theta)=2 m_{T} s .
$$

In particular, for every $(s, \theta) \in Q^{2}, \partial_{s} v(s, \theta)>0$.

We deduce from Lemma 3.3 that for every $(s, \theta) \in Q^{2}$,

$$
m_{\sigma} \frac{\mu^{2}}{2 \nu} s \leq g_{1}(s, \theta) \leq \frac{10 m_{\sigma} \nu^{2}}{\mu} s .
$$

Integrating with respect to $s$ those inequalities between 0 and $v(s, \theta)$, using (3.15), and the fact that $m_{\sigma} \mu \leq m_{T} \leq m_{\sigma} \nu$, we get

$$
\frac{\mu}{\sqrt{5} \nu} s \leq v(s, \theta) \leq 2 \frac{\nu}{\mu} s .
$$

From 3.16) and 3.14,

$$
0<v(s, \theta) \partial_{s} v(s, \theta) \leq 4 \frac{\nu^{2}}{\mu^{2}} s .
$$

In view of 3.17, this also implies that

$$
0<\partial_{s} v(s, \theta) \leq 4 \sqrt{5} \frac{\nu^{3}}{\mu^{3}} .
$$

A similar argument proves that $\partial_{\theta} v \in L^{\infty}\left(Q^{2}\right)$. We now define

$$
\phi_{0}(s, \theta)=(v(s, \theta), \theta) .
$$

Then $\phi_{0}$ is a homeomorphism from $\overline{Q^{2}}$ onto $\overline{Q^{2}}$ which is $C^{1}$ on $Q^{2}$. Moreover, $\phi_{0}(0, \theta)=$ $(0, \theta), \phi_{0}(1, \theta)=(1, \theta)$ and $\operatorname{Jac} \phi_{0}(s, \theta)=\partial_{s} v(s, \theta)$. By differentiation of 3.15), we get

$$
2 m_{T} s=g_{1}(v(s, \theta), \theta) \partial_{s} v(s, \theta)=g_{1} \circ \phi_{0}(s, \theta) \mathbf{J a c} \phi_{0}(s, \theta) .
$$


This completes the proof of the lemma.

Gathering the previous results we can finally conclude the proof.

Proof of Proposition 3.2

Lemmas 3.3. 3.4 clearly show that, as announced, the map

$$
\Phi=\phi_{2} \circ \phi_{1} \circ \phi_{0},
$$

satisfies all the assumptions of Proposition 3.1. The structure is summarized in Figure 3.1.

- The side $\{s=0\}$ of $Q^{2}$ (in red solid on the figure) is pointwise preserved by $\phi_{0}$ and $\phi_{1}$ and mapped to the vertex of $T$ (also in red) by $\phi_{2}$.

- The side $\{s=1\}$ of $Q^{2}$ (in blue dashdotted on the figure) is pointwise preserved by $\phi_{0}$ and $\phi_{1}$ and mapped to $\sigma$ by $\phi_{2}$.

- The horizontal segments $\{\theta=$ cte $\}$ of $Q^{2}$ (in magenta dashed) are preserved as a whole by $\phi_{0}$ then deformed by $\phi_{1}$ and $\phi_{2}$.

- The vertical segments $\{s=$ cte $\}$ (in green dotted) are preserved as a whole by $\phi_{1}$ and deformed by $\phi_{0}^{-1}$ and $\phi_{2}$.

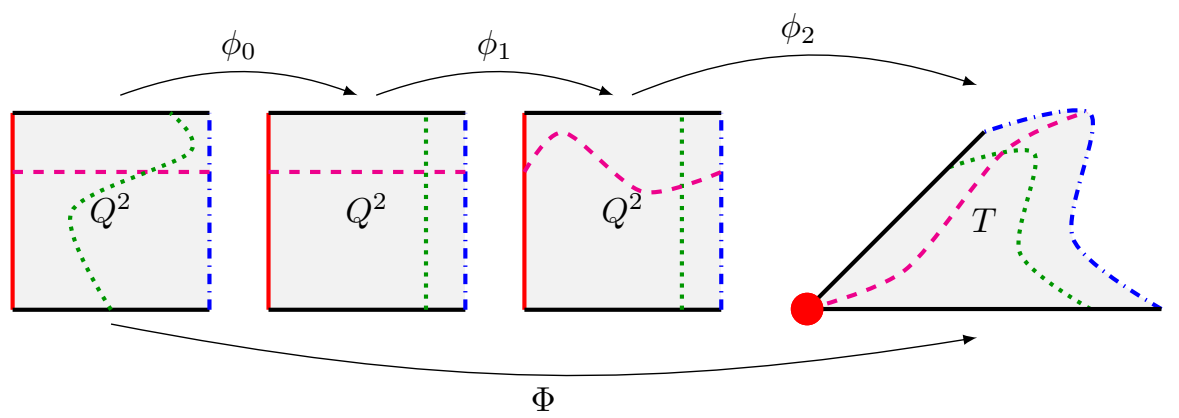

FIG. 3.1. Construction of the diffeomorphism $\Phi$

Moreover, by definition, we have (using the same notation as in the proofs of the above two lemmas)

$$
\Phi(s, \theta)=v(s, \theta) \gamma(\theta+\zeta \circ v(s, \theta) w(\theta)) .
$$

Hence,

$$
\begin{aligned}
\partial_{s} \Phi(s, \theta)= & \left(\partial_{s} v(s, \theta)\right) \gamma(\theta+\zeta \circ v(s, \theta) w(\theta)) \\
& +v(s, \theta) \partial_{s} v(s, \theta) \zeta^{\prime}(v(s, \theta)) w(\theta) \gamma^{\prime}(\theta+\zeta \circ v(s, \theta) w(\theta)) .
\end{aligned}
$$

By construction, $\left|\zeta^{\prime}\right|_{L^{\infty}} \leq 10 / \varepsilon=100 \nu / \mu$ and $|w|_{L^{\infty}} \leq 1$. It then follows from 3.18 and 3.19 ) that

$$
\left|\partial_{s} \Phi(s, \theta)\right| \leq C \frac{\nu^{3}}{\mu^{3}}\left(\operatorname{diam}(T)+m_{\sigma}\right) .
$$

The proof is complete.

4. A Poincaré inequality. In this section, we derive a Poincaré inequality related to Theorem 2.1

THEOREM 4.1. We consider the same assumption (2.2) as in Theorem 2.1] Then for every $p \in\left[1,+\infty\left[\right.\right.$ and every $u \in W^{1, p}(T)$,

$$
\frac{1}{m_{T}} \int_{T}\left|u-\frac{1}{m_{\sigma}} \int_{\sigma} u\right|^{p} \leq C\left(\operatorname{diam}(T)+m_{\sigma}\right)^{p} \frac{1}{m_{T}} \int_{T}|D u|^{p}
$$


where $C$ only depends on $p$ and on the ratio $\frac{\nu}{\mu}$.

This result is a consequence of the inequality proved in the previous section and of the following lemma whose proof is postponed at the end of the section.

LEMMA 4.2. Under the assumption (2.2), for every $u \in W^{1, p}(T)$, we have

$$
\frac{1}{m_{T}^{2}} \int_{T} \int_{T}\left|u(x)-u\left(x^{\prime}\right)\right|^{p} d x d x^{\prime} \leq C\left(\operatorname{diam}(T)+m_{\sigma}\right)^{p} \frac{1}{m_{T}} \int_{T}|D u|^{p},
$$

where $C$ only depends on $p$ and on the ratio $\frac{\nu}{\mu}$.

Proof of Theorem 4.1. By the triangle inequality,

$$
\frac{1}{m_{T}} \int_{T}\left|u-\frac{1}{m_{\sigma}} \int_{\sigma} u\right|^{p} \leq C_{p} \frac{1}{m_{T}} \int_{T}\left|u-\frac{1}{m_{T}} \int_{T} u\right|^{p}+C_{p}\left|\frac{1}{m_{T}} \int_{T} u-\frac{1}{m_{\sigma}} \int_{\sigma} u\right|^{p} .
$$

One can estimate the second term with Theorem 2.1.

$$
\left|\frac{1}{m_{T}} \int_{T} u-\frac{1}{m_{\sigma}} \int_{\sigma} u\right|^{p} \leq C^{p}\left(m_{\sigma}+\operatorname{diam}(T)\right)^{p} \frac{1}{m_{T}} \int_{T}|D u|^{p},
$$

where $C$ only depends on the ratio $\frac{\nu}{\mu}$. By Jensen's inequality, the first term is not larger than the quantity

$$
\frac{1}{m_{T}^{2}} \int_{T} \int_{T}\left|u(x)-u\left(x^{\prime}\right)\right|^{p} d x d x^{\prime},
$$

which is, in turn, estimated by using Lemma 4.2 .

It remains to prove the lemma.

Proof of Lemma 4.2. Let us introduce the reference unit triangle $T_{0}$ defined by

$$
T_{0}=\{(a, b) \in] 0,1\left[{ }^{2}, \quad b<a\right\} .
$$

On this domain, the following inequality classicaly holds

$$
\int_{T_{0}} \int_{T_{0}}\left|v(y)-v\left(y^{\prime}\right)\right|^{p} d y d y^{\prime} \leq C_{p} \int_{T_{0}}|D v(y)|^{p} d y, \quad \forall v \in W^{1, p}\left(T_{0}\right),
$$

with a value of $C_{p}>0$ depending only on $p$.

We introduce (see Figure 4.1) the following diffeomorphism from $T_{0}$ onto $T$

$$
\Psi:(a, b) \in T_{0} \mapsto a \gamma(b / a) \in T .
$$

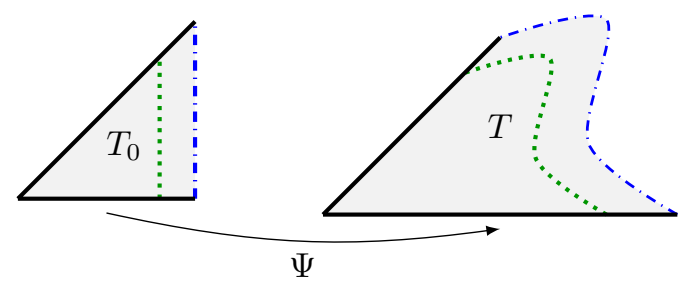

FIG. 4.1. The diffeomorphism $\Psi$

We proceed now with the estimate of the derivatives of $\Psi$. An immediate computation shows that

$$
D \Psi(a, b)=\left(\gamma(b / a)-(b / a) \gamma^{\prime}(b / a), \gamma^{\prime}(b / a)\right)
$$


so that we get

$$
\operatorname{Jac} \Psi(a, b)=\operatorname{det}\left(\gamma(b / a), \gamma^{\prime}(b / a)\right),
$$

and thus, by 2.4] (which is a consequence of (2.2)), we deduce that

$$
0<2 \mu m_{\sigma} \leq \operatorname{Jac} \Psi(a, b) \leq 2 \nu m_{\sigma}, \quad \forall(a, b) \in T_{0},
$$

and

$$
\|D \Psi\|_{\infty} \leq\left(\|\gamma\|_{\infty}+\left\|\gamma^{\prime}\right\|_{\infty}\right) \leq\left(\operatorname{diam}(T)+m_{\sigma}\right) .
$$

For any $u \in C^{1}(\bar{T})$ we set $v=u \circ \Psi \in W^{1, p}\left(T_{0}\right)$ and we use $\Psi$ as a change of variables

$$
\begin{aligned}
\int_{T} \int_{T}\left|u(x)-u\left(x^{\prime}\right)\right|^{p} d x d x^{\prime} & =\int_{T_{0}} \int_{T_{0}}\left|v(y)-v\left(y^{\prime}\right)\right|^{p} \operatorname{Jac} \Psi(y) \operatorname{Jac} \Psi\left(y^{\prime}\right) d y d y^{\prime} \\
& \leq\|\operatorname{Jac} \Psi\|_{\infty}^{2} \int_{T_{0}} \int_{T_{0}}\left|v(y)-v\left(y^{\prime}\right)\right|^{p} d y d y^{\prime} .
\end{aligned}
$$

Then by 4.1] and the change of variables $x=\Psi(y)$ again, we get

$$
\begin{aligned}
& \int_{T} \int_{T}\left|u(x)-u\left(x^{\prime}\right)\right|^{p} d x d x^{\prime} \leq C_{p}\|\operatorname{Jac} \Psi\|_{\infty}^{2} \int_{T_{0}}|D v(y)|^{p} d y
\end{aligned}
$$

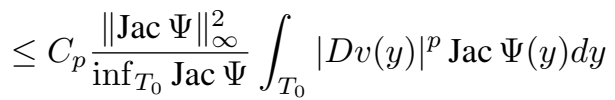

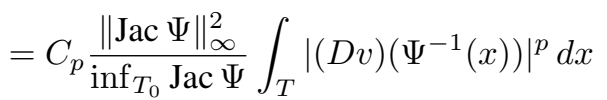

$$
\begin{aligned}
& =C_{p} \frac{\|\operatorname{Jac} \Psi\|_{\infty}^{2}}{\inf _{T_{0}} \operatorname{Jac} \Psi} \int_{T}|D u(x)|^{p}\left\|D \Psi\left(\Psi^{-1}(x)\right)\right\|^{p} d x \\
& \leq C_{p} \frac{\|\operatorname{Jac} \Psi\|_{\infty}^{2}\|D \Psi\|_{\infty}^{p}}{\inf _{T_{0}} \operatorname{Jac} \Psi} \int_{T}|D u(x)|^{p} d x .
\end{aligned}
$$

By using the previous estimates 4.2 and $(4.3$ we conclude that

$$
\begin{aligned}
\int_{T} \int_{T}\left|u(x)-u\left(x^{\prime}\right)\right|^{p} d x d x^{\prime} & \leq C_{p} \frac{2 m_{\sigma} \nu^{2}}{\mu}\left(\operatorname{diam}(T)+m_{\sigma}\right)^{p} \int_{T}|D u(x)|^{p} d x \\
& \leq C_{p} \frac{2 m_{\sigma} \nu^{2}}{\mu^{2}} \mu\left(\operatorname{diam}(T)+m_{\sigma}\right)^{p} \int_{T}|D u(x)|^{p} d x .
\end{aligned}
$$

Since $\mu m_{\sigma} \leq m_{T}$, we finally obtain

$$
\int_{T} \int_{T}\left|u(x)-u\left(x^{\prime}\right)\right|^{p} d x d x^{\prime} \leq C m_{T}\left(\operatorname{diam}(T)+m_{\sigma}\right)^{p} \int_{T}|D u(x)|^{p} d x
$$

for a $C$ depending only on $p$ and $\nu / \mu$. Dividing this inequality by $m_{T}^{2}$ gives the claim.

5. The higher dimensional case. Let $n \geq 2$. Let $\gamma: \overline{Q^{n-1}} \rightarrow \mathbb{R}^{n}$ be a $C^{1}$ map on the closure of the unit cube $Q^{n-1}=(0,1)^{n-1}$ such that $\gamma$ is one-to-one and $\left|\partial_{1} \gamma \wedge \cdots \wedge \partial_{n-1} \gamma\right|>$ 0 on $\overline{Q^{n-1}}$. We denote by $\sigma=\gamma\left(\overline{Q^{n-1}}\right)$ the corresponding hypersurface and by $T$ the set:

$$
T=\left\{s \gamma(\theta): s \in(0,1), \theta \in Q^{n-1}\right\} .
$$


We assume that for every $\theta \in \overline{Q^{n-1}}$,

$$
\{s \gamma(\theta), s \geq 0\} \cap \sigma=\{\gamma(\theta)\} .
$$

THEOREM 5.1. We assume that there exist $\mu, \nu>0$ such that for every $\theta \in Q^{n-1}$,

$$
\mu \leq \frac{\operatorname{det}\left(\gamma, \partial_{1} \gamma, \ldots, \partial_{n-1} \gamma\right)(\theta)}{n\left|\partial_{1} \gamma \wedge \cdots \wedge \partial_{n-1} \gamma\right|(\theta)} \leq \nu
$$

Then for every $p \in\left[1,+\infty\left[\right.\right.$ and every $u \in W^{1, p}(T)$,

$$
\left|\frac{1}{m_{\sigma}} \int_{\sigma} u-\frac{1}{m_{T}} \int_{T} u\right|^{p} \leq C^{p}\left(\|D \gamma\|_{L^{\infty}}+\operatorname{diam}(T)\right)^{p} \frac{1}{m_{T}} \int_{T}|D u|^{p},
$$

where $C$ only depends on the ratio $\frac{\nu}{\mu}$.

REMARK 5.1. Observe that the quantity in (5.1) is invariant with respect to the parametrization of $\sigma$. More precisely, let $\psi: Q^{n-1} \rightarrow Q^{n-1}$ be a $C^{1}$ map such that $\mid$ Jac $\psi \mid>0$ everywhere and $\widetilde{\gamma}=\gamma \circ \psi$. Then

$$
\partial_{1} \widetilde{\gamma} \wedge \cdots \wedge \partial_{n-1} \widetilde{\gamma}=\left(\left(\partial_{1} \gamma \wedge \cdots \wedge \partial_{n-1} \gamma\right) \circ \psi\right) \mathrm{Jac} \psi .
$$

This implies

$$
\begin{aligned}
\operatorname{det}\left(\widetilde{\gamma}, \partial_{1} \tilde{\gamma}, \ldots, \partial_{n-1} \widetilde{\gamma}\right) & =\left\langle\widetilde{\gamma}, \partial_{1} \widetilde{\gamma} \wedge \cdots \wedge \partial_{n-1} \tilde{\gamma}\right\rangle \\
& =\left\langle\widetilde{\gamma},\left(\left(\partial_{1} \gamma \wedge \cdots \wedge \partial_{n-1} \gamma\right) \circ \psi\right) \operatorname{Jac} \psi\right\rangle \\
& =\left(\operatorname{det}\left(\gamma, \partial_{1} \gamma, \ldots, \partial_{n-1} \gamma\right) \circ \psi\right) \operatorname{Jac} \psi
\end{aligned}
$$

Hence,

$$
\frac{\operatorname{det}\left(\widetilde{\gamma}, \partial_{1} \widetilde{\gamma}, \ldots, \partial_{n-1} \widetilde{\gamma}\right)}{\left|\partial_{1} \widetilde{\gamma} \wedge \cdots \wedge \partial_{n-1} \widetilde{\gamma}\right|}=\frac{\operatorname{det}\left(\gamma, \partial_{1} \gamma, \ldots, \partial_{n-1} \gamma\right) \circ \psi}{\left|\partial_{1} \gamma \wedge \cdots \wedge \partial_{n-1} \gamma\right| \circ \psi}
$$

In particular, when $\psi$ is a $C^{1}$ diffeomorphism, $\gamma$ satisfies (5.1) if and only if $\widetilde{\gamma}$ satisfies (5.1).

In view of Lemma A.1 given in the appendix, one can assume without loss of generality that

$$
\left|\partial_{1} \gamma \wedge \cdots \wedge \partial_{n-1} \gamma\right|(\theta)=m_{\sigma}, \quad \forall \theta \in Q^{n-1} .
$$

Exactly as in the 2 dimensional case, the proof of Theorem 5.1 is a consequence of the following two propositions.

Proposition 5.2. Assume that there exists a Lipschitz continuous map $\Phi:[0,1] \times$ $\overline{Q^{n-1}} \rightarrow \bar{T}$ such that

1. $\Phi$ is a $C^{1}$ diffeomorphism from $(0,1) \times Q^{n-1}$ onto $T$,

2. $\Phi(0, \theta)=(0,0)$,

3. $\Phi(1, \theta)=\gamma(\theta)$,

4. $\operatorname{Jac} \Phi(s, \theta)=n m_{T} s^{n-1}$.

Then for every $u \in W^{1,1}(T)$, we have

$$
\frac{1}{m_{\sigma}} \int_{\sigma} u-\frac{1}{m_{T}} \int_{T} u=\frac{1}{n m_{T}} \int_{T} D u(x, y)\left[s \partial_{s} \Phi(s, \theta)\right]_{(s, \theta)=\Phi^{-1}(x, y)} d x d y .
$$


The proof is very similar to the proof of Proposition 3.1 and we omit it. In particular, we observe that by (5.2), we have

$$
\begin{aligned}
\int_{\sigma} u=\int_{Q^{n-1}} u(\gamma(\theta))\left|\partial_{1} \gamma \wedge \cdots \wedge \partial_{n-1} \gamma\right|(\theta) d \theta & =m_{\sigma} \int_{Q^{n-1}} u(\gamma(\theta)) d \theta \\
& =m_{\sigma} \int_{Q^{n-1}} u(\Phi(1, \theta)) d \theta
\end{aligned}
$$

The construction of a suitable $\Phi$ then follows the lines of the two dimensional case. More precisely, the following proposition is the analogue of Proposition 3.2 .

PROPOSITION 5.3. There exists $a \Phi:[0,1] \times \overline{Q^{n-1}} \rightarrow \bar{T}$ satisfying the assumptions of Proposition 5.2 and such that

$$
\left|\partial_{s} \Phi(s, \theta)\right| \leq C\left(\operatorname{diam}(T)+\|D \gamma\|_{L^{\infty}}\right)
$$

where $C$ only depends on $\nu / \mu$.

Proof of Theorem 5.1. By Jensen's inequality, we only need to prove the case $p=1$ and by a standard approximation argument, one can further assume that $\gamma \in C^{2}\left(\overline{Q^{n-1}}\right)$. The required inequality is then a consequence of the equality given by Proposition 5.2, and of the construction and estimate of the map $\Phi$ given by Proposition 5.3 .

It remains to prove Proposition 5.3. As in the $2 \mathrm{D}$ case, we will look for $\Phi$ in the following form

$$
\Phi=\phi_{2} \circ \phi_{1} \circ \phi_{0}
$$

where we still denote by $\phi_{2}$ the map

$$
\phi_{2}:(s, \theta) \in[0,1] \times \overline{Q^{n-1}} \mapsto s \gamma(\theta) \in \bar{T} .
$$

The maps $\phi_{1}$ and $\phi_{0}$ are built in a similar way as in Section 3 so that we only proceed to indicate the major changes in the following two lemmas.

LEMMA 5.4. There exists a $C^{1}$ diffeomorphism $\phi_{1}:[0,1] \times \overline{Q^{n-1}} \rightarrow[0,1] \times \overline{Q^{n-1}}$ such that

1. for every $\theta \in Q^{n-1}, \phi_{1}(0, \theta)=(0, \theta)$ and $\phi_{1}(1, \theta)=(1, \theta)$,

2. for every $\theta \in Q^{n-1}$,

$$
\int_{0}^{1} \operatorname{Jac}\left(\phi_{2} \circ \phi_{1}\right)(s, \theta) d s=m_{T} .
$$

3. for every $s \in[0,1]$ and every $\theta \in \overline{Q^{n-1}}$,

$$
\left|\partial_{s} \phi_{1}(s, \theta)\right| \leq C \quad, \quad C^{\prime} \leq \operatorname{Jac} \phi_{1}(s, \theta) \leq C^{\prime \prime}
$$

Proof.

where $C, C^{\prime}$ and $C^{\prime \prime}>0$ only depend on the ratio $\nu / \mu$.

It is divided into three steps. In the first one we detail an auxiliary construction that will be used in Step 2 in order to build, by induction, the diffeomorphism $\phi_{1}$. In the third and final step, we establish the required estimates 5.3.

Step 1: Construction of an auxiliary function

Fix $1 \leq k \leq n-1$. Given $\theta \in \overline{Q^{n-1}}$, we use the notation $\theta^{k-1}=\left(\theta_{1}, \ldots, \theta_{k-1}\right)$ and $\theta^{\prime}=\left(\theta_{k+1}, \ldots, \theta_{n-1}\right)$. Assume that there exists a $C^{1}$ function $h:[0,1] \times \overline{Q^{n-1}} \rightarrow \mathbb{R}$ such that 
1. for every $\theta^{\prime}=\left(\theta_{k+1}, \ldots, \theta_{n-1}\right) \in \overline{Q^{n-k-1}}$,

$$
\int_{(0,1) \times Q^{k}} h\left(s, \theta^{k}, \theta^{\prime}\right) d s d \theta^{k}=m_{T},
$$

2. there exist $C<1<C^{\prime}$ only depending on $\nu / \mu$ such that

$$
C n m_{\sigma} \mu s^{n-1} \leq h(s, \theta) \leq C^{\prime} n m_{\sigma} \nu s^{n-1}, \forall(s, \theta) \in[0,1] \times \overline{Q^{n-1}} .
$$

We introduce

$$
\varepsilon=\frac{C \mu}{2 n C^{\prime} \nu}
$$

and a cut-off function: $\zeta \in C_{c}^{\infty}\left((0,1) \times Q^{k-1}\right)$ such that $0 \leq \zeta \leq 1+\varepsilon$ and

$$
\int_{(0,1) \times Q^{k-1}} \zeta\left(s, \theta^{k-1}\right) d s d \theta^{k-1}=1, \quad \int_{(0,1) \times Q^{k-1}}\left|\zeta\left(s, \theta^{k-1}\right)-1\right| d s d \theta^{k-1}<\varepsilon .
$$

We can further require that

$$
\|D \zeta\|_{L^{\infty}} \leq \frac{C_{n}}{\varepsilon}
$$

for some constant $C_{n}$ which only depends on $n$. Consider the map

$$
G_{\theta^{\prime}}:(a, b) \mapsto \int_{(0,1) \times Q^{k-1}} d s d \theta^{k-1} \int_{0}^{a+\zeta\left(s, \theta^{k-1}\right) b} h\left(s, \theta^{k}, \theta^{\prime}\right) d \theta_{k} .
$$

Then $G_{\theta^{\prime}}$ is well-defined and $C^{2}$ on the set $\left\{(a, b): 0 \leq a \leq 1, \frac{-a}{1+\varepsilon} \leq b \leq \frac{1-a}{1+\varepsilon}\right\}$. As in the proof of Lemma 3.3 there exists a $C^{2}$ map $w:[0,1] \times \overline{Q^{n-k-1}} \mapsto[-1,1]$ such that for every $a \in[0,1]$ and every $\theta^{\prime} \in \overline{Q^{n-k-1}}, G_{\theta^{\prime}}\left(a, w\left(a, \theta^{\prime}\right)\right)=m_{T} a$; that is,

$$
\int_{(0,1) \times Q^{k-1}} d s d \theta^{k-1} \int_{0}^{a+\zeta\left(s, \theta^{k-1}\right) w\left(a, \theta^{\prime}\right)} h\left(s, \theta^{k}, \theta^{\prime}\right) d \theta_{k}=m_{T} a .
$$

Moreover, $w\left(0, \theta^{\prime}\right)=0=w\left(1, \theta^{\prime}\right)$ and by differentiation of the above identity with respect to $a$, one gets

$$
\begin{aligned}
\int_{(0,1) \times Q^{k-1}} h\left(s, \theta^{k-1}, a+\zeta\left(s, \theta^{k-1}\right) w\left(a, \theta^{\prime}\right), \theta^{\prime}\right) \\
\left(1+\zeta\left(s, \theta^{k-1}\right) \partial_{a} w\left(a, \theta^{\prime}\right)\right) d s d \theta^{k-1}=m_{T} .
\end{aligned}
$$

As in the proof of Lemma 3.3 , this leads to the following estimate:

$$
D \leq 1+\zeta\left(s, \theta^{k-1}\right) \partial_{a} w\left(a, \theta^{\prime}\right) \leq D^{\prime}
$$

for some constants $D, D^{\prime}>0$ which only depend on $\nu / \mu$. We omit the details.

\section{Step 2 : construction of $\phi_{1}$}

Let $\psi_{n+1}=i d_{[0,1] \times \overline{Q^{n-1}}}$ and $h_{n+1}=h_{n}=\operatorname{Jac} \phi_{2}$. We construct by induction on $k=n, \ldots, 0$ two sequences of maps

$$
\psi_{k+1}:[0,1] \times \overline{Q^{n-1}} \rightarrow[0,1] \times \overline{Q^{n-1}} \quad, \quad h_{k}:[0,1] \times \overline{Q^{n-1}} \rightarrow \mathbb{R}
$$

such that for $k=0, \ldots, n$, 
1. The map $\psi_{k+1}$ is a $C^{2}$ diffeomorphism from $[0,1] \times \overline{Q^{n-1}}$ onto $[0,1] \times \overline{Q^{n-1}}$,

2. For every $\theta \in Q^{n-1}, \psi_{k+1}(0, \theta)=(0, \theta)$ and $\psi_{k+1}(1, \theta)=(1, \theta)$,

3. We have

$$
h_{k}=\left(h_{k+1} \circ \psi_{k+1}\right) \mathbf{J a c} \psi_{k+1},
$$

4. There exist two constants $0<C_{k}<1<C_{k}^{\prime}$ depending only on $\nu / \mu$ such that for every $s \in[0,1]$ and every $\theta \in \overline{Q^{n-1}}$,

$$
\begin{gathered}
C_{k} \leq \mathrm{Jac} \psi_{k+1}(s, \theta) \leq C_{k}^{\prime}, \\
C_{k} n m_{\sigma} \mu s^{n-1} \leq h_{k}(s, \theta) \leq C_{k}^{\prime} n m_{\sigma} \nu s^{n-1} .
\end{gathered}
$$

5. We have

$$
\int_{(0,1) \times Q^{k}} h_{k}\left(s, \theta^{k}, \theta^{\prime}\right) d s d \theta^{k}=m_{T},
$$

where $\theta^{k}=\left(\theta_{1}, \ldots, \theta_{k}\right)$ and $\theta^{\prime}=\left(\theta_{k+1}, \ldots, \theta_{n-1}\right)$.

Observe that these conditions are satisfied for $k=n$. We assume that for some $k \geq 1$, $\psi_{n+1}, \psi_{n} \ldots, \psi_{k+1}$, and thus $h_{n}, \ldots, h_{k}$ are already constructed and satisfy the above properties. Let $\varepsilon_{k}=\frac{C_{k} \mu}{2 n C_{k}^{\prime} \nu}$ and $\zeta_{k}$ a function which satisfies the properties of the function $\zeta$ introduced in Step 1, with $\varepsilon=\varepsilon_{k}$. We apply Step 1 to the function $h_{k}$ with $\zeta_{k}$ and $C=C_{k}$, $C^{\prime}=C_{k}^{\prime}$. This gives a function $w_{k}:[0,1] \times \overline{Q^{n-k-1}} \rightarrow[-1,1]$ satisfying the properties enumerated in Step 1.

We then construct $\psi_{k}$ as follows

$$
\begin{aligned}
\psi_{k}\left(s, \theta_{1}, \ldots, \theta_{n-1}\right) & =\left(s, \theta^{k-1}, v_{k}(s, \theta), \theta^{\prime}\right) \\
& =\left(s, \theta_{1}, \ldots, \theta_{k-1}, v_{k}(s, \theta), \theta_{k+1}, \ldots, \theta_{n-1}\right)
\end{aligned}
$$

with

$$
v_{k}(s, \theta)=\theta_{k}+\zeta_{k}\left(s, \theta^{k-1}\right) w_{k}\left(\theta_{k}, \theta^{\prime}\right) .
$$

Since Jac $\psi_{k}=1+\zeta_{k} \partial_{\theta_{k}} w_{k}, 5.5$ implies

$$
C_{k-1} \leq \operatorname{Jac} \psi_{k} \leq C_{k-1}^{\prime},
$$

where $0<C_{k-1}<1<C_{k-1}^{\prime}$ only depend on $\nu / \mu$.

Let $h_{k-1}=\left(h_{k} \circ \psi_{k}\right) \mathrm{Jac} \psi_{k}$. By (5.4),

$$
\begin{aligned}
\int_{(0,1) \times Q^{k-1}} & h_{k-1} \\
= & \int_{(0,1) \times Q^{k-1}} h_{k}\left(s, \theta^{k-1}, \theta_{k}+\zeta_{k}\left(s, \theta^{k-1}\right) w_{k}\left(\theta_{k}, \theta^{\prime}\right), \theta^{\prime}\right) \mathbf{J a c} \psi_{k}(s, \theta) d s d \theta^{k-1} \\
= & m_{T} .
\end{aligned}
$$

As in the proof of Lemma 3.3, one can check that $h_{k-1}$ and $\psi_{k}$ satisfy all the remaining properties, even if it means changing the actual value of the constants $C_{k-1}$ and $C_{k-1}^{\prime}$. This completes the construction by induction of $h_{0}, \ldots, h_{n}$ and $\psi_{1}, \ldots, \psi_{n+1}$. We now define

$$
\phi_{1}=\psi_{n} \circ \psi_{n-1} \circ \cdots \circ \psi_{1} .
$$


Then $\phi_{1}$ is a $C^{1}$ diffeomorphism from $[0,1] \times \overline{Q^{n-1}}$ onto itself and $\phi_{1}$ coincides with the identity on $\{0\} \times \overline{Q^{n-1}}$ and $\{1\} \times \overline{Q^{n-1}}$. By construction,

$$
\begin{aligned}
h_{0}=\left(h_{1} \circ \psi_{1}\right) \operatorname{Jac} \psi_{1} & =\left(\left(\left(h_{2} \circ \psi_{2}\right) \mathbf{J a c} \psi_{2}\right) \circ \psi_{1}\right) \mathbf{J a c} \psi_{1} \\
& =\left(h_{2} \circ \psi_{2} \circ \psi_{1}\right) \operatorname{Jac}\left(\psi_{2} \circ \psi_{1}\right)=\ldots \\
& =\left(h_{n} \circ \psi_{n} \circ \cdots \circ \psi_{1}\right) \operatorname{Jac}\left(\psi_{n} \circ \cdots \circ \psi_{1}\right) \\
& =\left(h_{n} \circ \phi_{1}\right) \operatorname{Jac} \phi_{1} \\
& =\left(\operatorname{Jac} \phi_{2} \circ \phi_{1}\right) \operatorname{Jac} \phi_{1}=\operatorname{Jac}\left(\phi_{2} \circ \phi_{1}\right) .
\end{aligned}
$$

In particular, we deduce that

$$
m_{T}=\int_{(0,1)} h_{0}\left(s, \theta^{\prime}\right) d s=\int_{(0,1)} \operatorname{Jac}\left(\phi_{2} \circ \phi_{1}\right)\left(s, \theta^{\prime}\right) d s .
$$

Step 3: Proof of (5.3)

For every $k=1, \ldots, n-1$, and every $(s, \theta) \in[0,1] \times \overline{Q^{n-1}}$, let us introduce the notation:

$$
[(s, \theta)]^{k}=\theta^{k}=\left(\theta_{1}, \ldots, \theta_{k}\right)
$$

Then

$$
\psi_{1}(s, \theta)=\left(s,\left[\psi_{1}(s, \theta)\right]^{1}, \theta^{\prime}\right)=\left(s, \theta_{1}+\zeta_{1}(s) w_{1}\left(\theta_{1}, \theta^{\prime}\right), \theta^{\prime}\right)
$$

with $\theta^{\prime}=\left(\theta_{2}, \ldots, \theta_{n-1}\right)$, and for every $k=1, \ldots, n$,

$$
\begin{aligned}
& \psi_{k} \circ \psi_{k-1} \circ \cdots \circ \psi_{1}(s, \theta) \\
& =\left(s,\left[\psi_{k-1} \circ \cdots \circ \psi_{1}(s, \theta)\right]^{k-1}, \theta_{k}+\zeta_{k}\left(s,\left[\psi_{k-1} \circ \cdots \circ \psi_{1}(s, \theta)\right]^{k-1}\right) w_{k}\left(\theta_{k}, \theta^{\prime}\right), \theta^{\prime}\right)
\end{aligned}
$$

where $\theta^{\prime}=\left(\theta_{k+1}, \ldots, \theta_{n}\right)$.

Since $\left\|w_{k}\right\|_{L^{\infty}} \leq 1$, it follows by induction on $k=1, \ldots, n$ that there exists $A_{k}>0$ which depends only on $\nu / \mu$ such that

$$
\left\|\partial_{s}\left(\psi_{k} \circ \cdots \circ \psi_{1}\right)\right\|_{L^{\infty}} \leq A_{k} .
$$

In particular, $\left\|\partial_{s} \phi_{1}\right\|_{L^{\infty}} \leq A_{n}$. The fact that $C \leq \mathrm{Jac} \phi_{1} \leq C^{\prime}$, for some $C, C^{\prime}>0$ depending only on $\nu / \mu$, follows from (5.6) and the identity

$$
\mathrm{Jac} \phi_{1}=\prod_{k=1}^{n} \mathrm{Jac} \psi_{k}
$$

The lemma is proved.

LEMMA 5.5. There exists an homeomorphism $\phi_{0}:[0,1] \times \overline{Q^{n-1}} \rightarrow[0,1] \times \overline{Q^{n-1}}$ which is $C^{1}$ on $(0,1) \times Q^{n-1}$ and such that

1. for every $\theta \in(0,1), \phi_{0}(0, \theta)=(0, \theta)$ and $\phi_{0}(1, \theta)=(1, \theta)$,

2. for every $(s, \theta) \in(0,1) \times Q^{n-1}$,

$$
\operatorname{Jac}\left(\phi_{2} \circ \phi_{1} \circ \phi_{0}\right)(s, \theta)=n m_{T} s^{n-1},
$$

3. there exists a $C^{1}$ map $v:(0,1) \times Q^{n-1} \rightarrow[0,1]$ such that for every $(s, \theta) \in$ $(0,1) \times Q^{n-1}, \phi_{0}(s, \theta)=(v(s, \theta), \theta)$ and

$$
|v(s, \theta)| \leq C s \quad, \quad\left|\partial_{s} v(s, \theta)\right| \leq C
$$

where $C$ only depends on $\nu / \mu$. 
The proof is essentially the same as the proof of Lemma 3.4 The only difference is that now by (5.6) for $k=0$ and (5.7)

$$
C n m_{\sigma} \mu s^{n-1} \leq \mathrm{Jac}\left(\phi_{2} \circ \phi_{1}\right) \leq C^{\prime} n m_{\sigma} \nu s^{n-1} .
$$

The rest of the proof is the same and we omit it.

Proof of Proposition 5.3. By construction,

$$
\Phi(s, \theta)=\phi_{2} \circ \phi_{1} \circ \phi_{0}(s, \theta)=\phi_{2}\left(\phi_{1}(v(s, \theta), \theta)\right) .
$$

Hence,

$$
\partial_{s} \Phi(s, \theta)=D \phi_{2}\left(\phi_{1}(v(s, \theta), \theta)\right) . \partial_{s} \phi_{1}(v(s, \theta), \theta) . \partial_{s} v(s, \theta) .
$$

By Lemma 5.5, $\left|\partial_{s} v\right|,|v| \leq C$ and by Lemma 5.4, we have $\left|\partial_{s} \phi_{1}\right| \leq C$. Hence

$$
\left|\partial_{s} \Phi(s, \theta)\right| \leq C\left(\operatorname{diam}(T)+\|D \gamma\|_{L^{\infty}}\right)
$$

where $C$ only depends on $\nu / \mu$.

\section{Applications to the analysis of some finite volume methods.}

6.1. Regular families of meshes of a smooth domain. Let $\Omega$ be a bounded domain of $\mathbb{R}^{2}$ with a $C^{2}$ boundary; we set $\Gamma=\partial \Omega$. A finite volume mesh $\mathfrak{M}$ of $\Omega$ is a finite family of compact subsets of $\bar{\Omega}$ with non-empty interiors usually refered to as control volumes and denoted by the letter $\mathcal{K}$. This family is supposed to satisfy

$$
\begin{gathered}
\stackrel{\mathcal{K}}{\mathcal{L}}=\emptyset, \quad \forall \mathcal{K}, \mathcal{L} \in \mathfrak{M}, \mathcal{K} \neq \mathcal{L}, \\
\bar{\Omega}=\bigcup_{\mathcal{K} \in \mathfrak{M}} \mathcal{K} .
\end{gathered}
$$

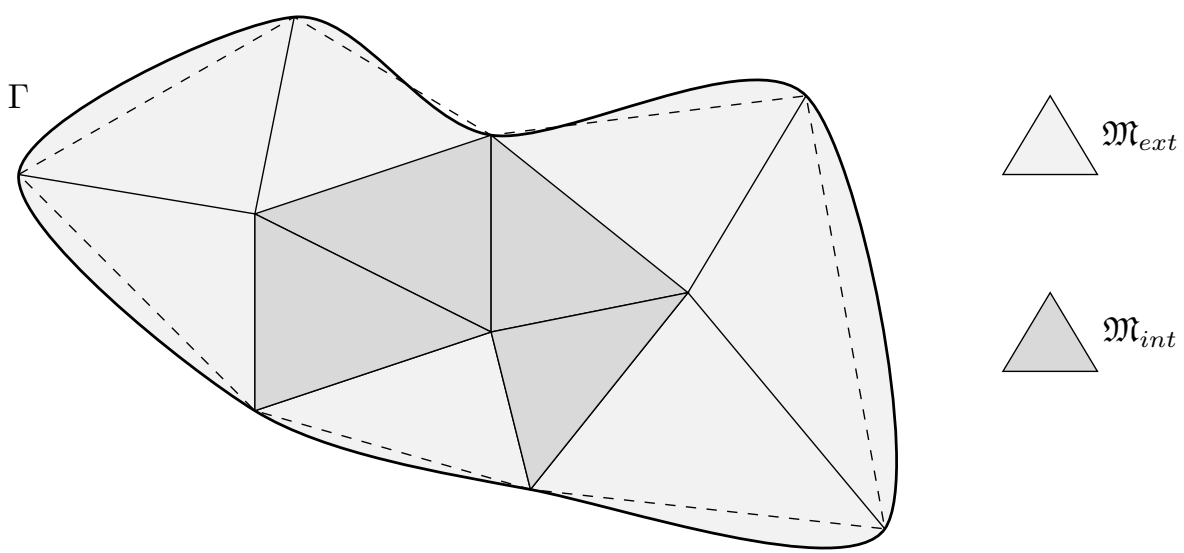

FIG. 6.1. The non-polygonal mesh $\mathfrak{M}$ of $\Omega$ and the two submeshes $\mathfrak{M}_{\text {int }}$ and $\mathfrak{M}_{\text {ext }}$

We assume that $\mathfrak{M}$ can be split into two disjoint subsets (see Figure 6.1) as follows:

- The set of polygonal control volumes $\mathfrak{M}_{\text {int }}$ that satisfy: for any $\mathcal{K} \in \mathfrak{M}_{\text {int }}, \mathcal{K}$ is polygonal and $\mathcal{K} \cap \partial \Omega$ contains at most a finite number of points. 
- The set of curved control volumes $\mathfrak{M}_{\text {ext }}$ that satisfy : for any $\mathcal{K} \in \mathfrak{M}_{\text {ext }}, \mathcal{K}$ is a pseudo-triangle whose curved edge is contained in the boundary of the domain $\Omega$. With any such curved control volume $\mathcal{K}$, we associate the (real) triangle $\widetilde{\mathcal{K}}$ which possesses the same vertices as $\mathcal{K}$ (see the dashed lines in Figure 6.1). Observe that $\widetilde{\mathcal{K}}$ may not be included in $\bar{\Omega}$.

We may now define the approximate mesh to be the following set of control volumes

$$
\widetilde{\mathfrak{M}}=\bigcup_{\mathcal{K} \in \mathfrak{M}_{\text {int }}}\{\mathcal{K}\} \cup \bigcup_{\mathcal{K} \in \mathfrak{M}_{\text {ext }}}\{\widetilde{\mathcal{K}}\} .
$$

This is a finite volume mesh made of polygonal control volumes.

The size and the regularity of such a mesh are measured by the quantities

$$
\operatorname{size}(\mathfrak{M})=\max _{\sigma \in \mathcal{E}} m_{\sigma}, \text { and } \operatorname{reg}_{1}(\mathfrak{M})=\max _{\mathcal{L} \in \mathfrak{M}} \frac{\operatorname{diam}(\mathcal{L})^{2}}{m_{\mathcal{L}}},
$$

where $\mathcal{E}$ is the set of the edges $\sigma$ of all the control volumes in the mesh $\mathfrak{M}$. Usual convergence results in the finite volume framework assume that $\operatorname{size}(\mathfrak{M})$ goes to 0 and that $\operatorname{reg}_{1}(\mathfrak{M})$ remains bounded. This means that control volumes are not allowed to become flat while the mesh is refined.

The main objective of this section is to prove that, if one builds a mesh $\mathfrak{M}$ of $\Omega$ as described previously such that $\operatorname{size}(\mathfrak{M})$ is small enough, then each boundary curved control volumes $\mathcal{K} \in \mathfrak{M}_{\text {ext }}$ satisfies the assumptions of Theorem 2.1 with a ratio $\nu_{\mathcal{K}} / \mu_{\mathcal{K}}$ which is independent of $\mathcal{K}$. In other words, on such curved elements, the inequality 2.3. holds with a constant $C$ uniformly bounded as the mesh is refined.

Proposition 6.1. Let $\Omega$ be a bounded domain of class $C^{2}$ in $\mathbb{R}^{2}$ and $\xi_{0}>0$. There exists $h_{0}>0$ depending only on $\Omega$ and $\xi_{0}$, such that for any finite volume mesh $\mathfrak{M}$ as described above, if

$$
\operatorname{reg}_{1}(\mathfrak{M}) \leq \xi_{0} \text { and } \operatorname{size}(\mathfrak{M}) \leq h_{0},
$$

then any exterior control volume $\mathcal{K} \in \mathfrak{M}_{\text {ext }}$ (which is a pseudo-triangle) satisfies the assumption (2.4) with two values of $\mu$ and $\nu$ that satisfy $\nu / \mu=3$.

Proof. The exterior control volume $\mathcal{K}$ can be written in the following form (see Figure 6.2

$$
\mathcal{K}=\{(1-s) \gamma(t): s \in[0,1], t \in[0, h]\},
$$

where the opposite vertex which is supposed to be the origin $(0,0)$ lies inside $\Omega$, and $\gamma$ : $[0, h] \rightarrow \mathbb{R}^{2}$ is a normal parametrization of the curved edge $\sigma \subset \Gamma:\left\|\gamma^{\prime}(t)\right\|=1$ for every $t \in[0, h]$.

We also introduce the associated real triangle $\widetilde{\mathcal{K}}$ with vertices $(0,0), \gamma(0)$ and $\gamma(h)$.

First, we claim that if we assume that

$$
h_{0}<\frac{1}{2\left\|\gamma^{\prime \prime}\right\|_{\infty}},
$$

then we have

$$
m_{\sigma}=h \leq 2 \operatorname{diam}(\widetilde{\mathcal{K}}) .
$$

Indeed, let $t \in[0, h]$. By the mean value inequality, there exists $\xi_{t} \in[0, h]$ such that

$$
\begin{aligned}
\left\langle\gamma^{\prime}(t), \gamma(h)-\gamma(0)\right\rangle & =h\left\langle\gamma^{\prime}(t), \gamma^{\prime}\left(\xi_{t}\right)\right\rangle \\
& \geq h-h^{2}\left\|\gamma^{\prime}\right\|_{\infty}\left\|\gamma^{\prime \prime}\right\|_{\infty}=h-h^{2}\left\|\gamma^{\prime \prime}\right\|_{\infty} \\
& \geq \frac{h}{2} .
\end{aligned}
$$




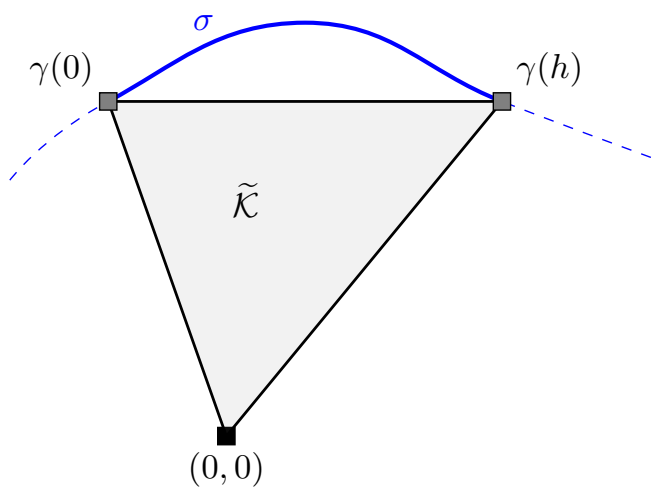

FIG. 6.2. A control volume $\mathcal{K} \in \mathfrak{M}_{\text {ext }}$ with a curved edge $\sigma \subset \Gamma$

The last inequality follows from (6.1) and 6.2). The conclusion follows from the CauchySchwarz inequality and the fact that the parametrization $\gamma$ is normal and satisfies $\| \gamma(h)-$ $\gamma(0) \| \leq \operatorname{diam}(\widetilde{\mathcal{K}})$.

Then, we are going to prove relation (2.4). For any $t \in[0, h]$, we write the term $\operatorname{det}\left(\gamma(t), \gamma^{\prime}(t)\right)$ as follows:

$$
\begin{aligned}
\operatorname{det}\left(\gamma(t), \gamma^{\prime}(t)\right)= & \operatorname{det}\left(\gamma(0), \frac{\gamma(h)-\gamma(0)}{h}\right) \\
& +\operatorname{det}\left(\gamma(0), \gamma^{\prime}(t)-\frac{\gamma(h)-\gamma(0)}{h}\right) \\
& +\operatorname{det}\left(\gamma(t)-\gamma(0), \gamma^{\prime}(t)\right) \\
:= & I_{1}+I_{2}+I_{3} .
\end{aligned}
$$

Now, we have to control the terms $I_{j}, j=1,2,3$.

We begin with the term $I_{1}$. Clearly, we have

$$
I_{1}=\frac{2}{h} m_{\widetilde{\mathcal{K}}}
$$

As regards the second term in [6.4, there exists $\zeta_{t} \in[0, h]$ such that,

$$
\left|I_{2}\right|=\left|\operatorname{det}\left(\gamma(0), \gamma^{\prime}(t)-\gamma^{\prime}\left(\zeta_{t}\right)\right)\right| \leq\|\gamma(0)\|\left\|\gamma^{\prime \prime}\right\|_{\infty} h \leq 2\left\|\gamma^{\prime \prime}\right\|_{\infty} \operatorname{diam}(\widetilde{\mathcal{K}})^{2} .
$$

Now, we are concerned by the last term in 6.4]. There exists $\widetilde{\zeta}_{t} \in[0, h]$ such that,

$$
\left|I_{3}\right|=\left|\operatorname{det}\left(-t \gamma^{\prime}(t)+\frac{t^{2}}{2} \gamma^{\prime \prime}\left(\widetilde{\zeta}_{t}\right), \gamma^{\prime}(t)\right)\right| \leq \frac{h^{2}}{2}\left\|\gamma^{\prime}\right\|_{\infty}\left\|\gamma^{\prime \prime}\right\|_{\infty}
$$

and since the parametrization is normal, we deduce by 6.3 that

$$
\left|I_{3}\right| \leq \frac{h^{2}}{2}\left\|\gamma^{\prime}\right\|_{\infty}\left\|\gamma^{\prime \prime}\right\|_{\infty} \leq 2\left\|\gamma^{\prime \prime}\right\|_{\infty}(\operatorname{diam}(\widetilde{\mathcal{K}}))^{2} .
$$

Gathering relations 6.4 -6.7) we get,

$$
\left|\frac{2}{h} m_{\widetilde{\mathcal{K}}}-\operatorname{det}\left(\gamma(t), \gamma^{\prime}(t)\right)\right| \leq 4\left\|\gamma^{\prime \prime}\right\|_{\infty}(\operatorname{diam}(\widetilde{\mathcal{K}}))^{2} .
$$


Thanks to the definition of $\operatorname{reg}_{1}(\mathfrak{M})$ and assumption 6.1 on $\operatorname{reg}_{1}(\mathfrak{M})$ we have,

$$
\left|\operatorname{det}\left(\gamma(t), \gamma^{\prime}(t)\right)-\frac{2}{h} m_{\widetilde{\mathcal{K}}}\right| \leq 4\left\|\gamma^{\prime \prime}\right\|_{\infty} \xi_{0} m_{\widetilde{\mathcal{K}}} \text {. }
$$

Then, we obtain

$$
m_{\widetilde{\mathcal{K}}}\left(\frac{2}{h}-4\left\|\gamma^{\prime \prime}\right\|_{\infty} \xi_{0}\right) \leq \operatorname{det}\left(\gamma(t), \gamma^{\prime}(t)\right) \leq m_{\widetilde{\mathcal{K}}}\left(\frac{2}{h}+4\left\|\gamma^{\prime \prime}\right\|_{\infty} \xi_{0}\right) .
$$

Assuming that $h_{0}$ satisfies, additionally to 6.2, the condition

$$
h_{0}<\frac{1}{4\left\|\gamma^{\prime \prime}\right\|_{\infty} \xi_{0}}
$$

we finally proved

$$
\frac{1}{h} m_{\widetilde{\mathcal{K}}} \leq \operatorname{det}\left(\gamma(t), \gamma^{\prime}(t)\right) \leq \frac{3}{h} m_{\widetilde{\mathcal{K}}}
$$

which exactly gives (2.4) with a ratio $\nu / \mu$ equal to 3 (observe that in (2.4), the parametrization is not normal but satisfies $\left|\gamma^{\prime}\right|=m_{\sigma}$ which does not change anything to the ratio $\left.\nu / \mu\right)$. The claim is proved provided one chooses a $h_{0}$ that satisfies 6.2 and 6.8 .

6.2. Example of application : the heat equation with dynamic Ventcell boundary conditions. As an illustration of the previous discussion we shall briefly describe a finite volume approximation of the following model problem

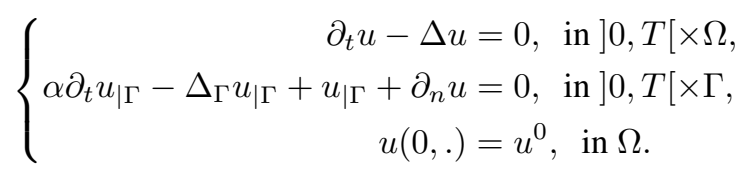

Here, $\alpha \geq 0$ is a parameter, $u_{\mid \Gamma}$ denotes the trace of $u$ on the boundary $\Gamma=\partial \Omega$ and $\Delta_{\Gamma}$ denotes the Laplace-Beltrami operator on $\Gamma$.

REMARK 6.1. The second equation of this system has to be understood as a boundary condition associated with the heat equation. It is usually refered to as a (dynamic, if $\alpha>0)$ Ventcell boundary condition, see for instance [10] for a recent work on this kind of problem.

We also refer to [9] where the result of the present paper was used as an important tool to give a complete convergence result (and as by-product a well-posedness result) for a much more complex model. This model is known as the Cahn-Hilliard equation with dynamic boundary condition. It is a fourth-order non-linear parabolic equation assorted with a nonlinear dynamic boundary condition.

The natural energy space for the problem 6.9 ) is the space

$$
H_{\Gamma}^{1}=\left\{u \in H^{1}(\Omega), u_{\mid \Gamma} \in H^{1}(\Gamma)\right\}
$$

endowed with the norm

$$
\|u\|_{H_{\Gamma}^{1}}=\left(\|\nabla u\|_{L^{2}(\Omega)}^{2}+\left\|u_{\mid \Gamma}\right\|_{L^{2}(\Gamma)}^{2}+\left\|\nabla_{\Gamma} u_{\mid \Gamma}\right\|_{L^{2}}^{2}\right)^{\frac{1}{2}}
$$

where $\nabla_{\Gamma}$ denotes the tangential gradient on $\Gamma$. 
A well-posedness result can be proved in this space, the main ingredient being the following formal energy estimate, obtained by multiplying the first equation by $\partial_{t} u$ and the boundary condition by $\partial_{t} u_{\mid \Gamma}$

$$
\begin{aligned}
\frac{d}{d t}\|u(t)\|_{H_{\Gamma}^{1}}^{2} & =\frac{d}{d t}\left(\int_{\Omega}|\nabla u(t, .)|^{2}+\int_{\Gamma}\left|u_{\mid \Gamma}(t, .)\right|^{2}+\int_{\Gamma}\left|\nabla_{\Gamma} u_{\mid \Gamma}(t, .)\right|^{2}\right) \\
& =-2 \int_{\Omega}\left|\partial_{t} u\right|^{2}-2 \alpha \int_{\Gamma}\left|\partial_{t} u_{\mid \Gamma}\right|^{2} .
\end{aligned}
$$

Let $\mathfrak{M}$ be a finite volume mesh of $\Omega$ as defined in Section 6.1. We recall here the main notations of the mesh $\mathfrak{M}$ (see Figure 6.3) used to obtain the finite volume scheme and we refer the reader to [7], for example, for more details.

We decompose $\mathcal{E}$ (the set of all the edges in the mesh) into the subset of exterior edges $\mathcal{E}_{\text {ext }}=\{\sigma \in \mathcal{E}: \sigma \subset \Gamma\}$ and the subset of interior edges $\mathcal{E}_{\text {int }}=\{\sigma \in \mathcal{E}: \sigma \not \subset \Gamma\}$. Similarly we use the notations $\mathcal{E}_{\mathcal{K}}^{\text {int }}$ and $\mathcal{E}_{\mathcal{K}}^{\text {ext }}$ for the edges of a given control volume $\mathcal{K} \in \mathfrak{M}$. If $\sigma$ is an interior edge which separates the control volumes $\mathcal{K}$ and $\mathcal{L}$, we note $\sigma=\mathcal{K} \mid \mathcal{L}$. For any neighboring exterior edges $\sigma, \tilde{\sigma} \in \mathcal{E}_{\text {ext }}$, we note $\mathbf{v}=\sigma \mid \tilde{\sigma}$ their common vertex (that belongs to $\Gamma$ ).

Let us remark that we have to solve an equation on the boundary $\Gamma$, thus we have to define boundary unknowns. In this context, we define a boundary mesh $\partial \mathfrak{M}$ which is in fact equal to the set of exterior edges of the initial mesh $\mathfrak{M}$. Thus, when we want to refer to the set of exterior edges we will note $\mathcal{E}_{\text {ext }}$ and when we want to refer to the set of boundary control volumes we will note $\partial \mathfrak{M}$. At each control volume $\mathcal{K} \in \mathfrak{M}$ we associate a point $x_{\mathcal{K}} \in \mathcal{K}$ called the center of the control volume $\mathcal{K}$ and at each edge $\sigma \in \mathcal{E}$ we associate a center $x_{\sigma} \in \sigma$. We assume that they satisfy the following orthogonality condition:

$$
\begin{aligned}
& {\left[x_{\mathcal{K}}, x_{\mathcal{L}}\right] \perp \sigma, \text { and } x_{\sigma}=\left[x_{\mathcal{K}}, x_{\mathcal{L}}\right] \cap \sigma, \quad \forall \sigma=\mathcal{K} \mid \mathcal{L} \in \mathcal{E}_{\text {int }},} \\
& {\left[x_{\mathcal{K}}, x_{\sigma}\right] \perp e_{\sigma}, \forall \sigma \in \mathcal{E}_{\mathcal{K}}^{\text {ext }}, \mathcal{K} \in \mathfrak{M}_{\text {ext }},}
\end{aligned}
$$

where $e_{\sigma}$ is the chord associated with $\sigma$ in the second case.

For $\mathcal{K} \in \mathfrak{M}$ and any edge $\sigma \in \mathcal{E}_{\mathcal{K}}$, we note $d_{\mathcal{K}, \sigma}$ the distance between the center $x_{\mathcal{K}}$ and the center $x_{\sigma}$, and for interior edges $\sigma=\mathcal{K} \mid \mathcal{L} \in \mathcal{E}_{\text {int }}$, we set $d_{\mathcal{K}, \mathcal{L}}=d_{\mathcal{K}, \sigma}+d_{\mathcal{L}, \sigma}$.

For any vertex $\mathbf{v}=\sigma \mid \tilde{\sigma}$, we define $d_{\sigma, \tilde{\sigma}}$ as the length of the arc included in $\Gamma$ whose ends are $x_{\sigma}, x_{\tilde{\sigma}}$ and passing through $\mathbf{v}=\sigma \mid \tilde{\sigma}$ (drawn with larger dashes on figure 6.3).

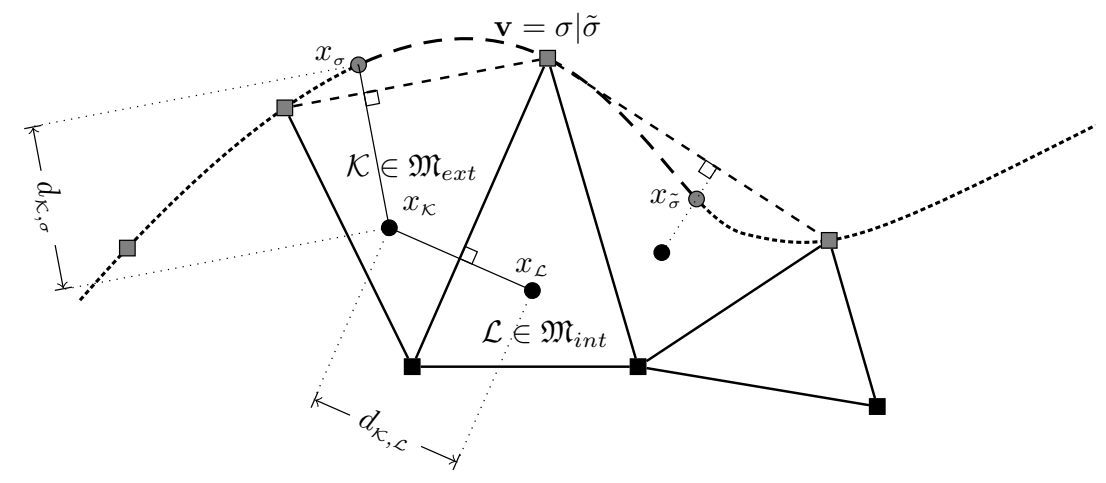

FIG. 6.3. Finite volume mesh $\mathfrak{M}$ of $\Omega$ 
With these new notations, we can now measure the regularity of the mesh with respect to the position of the centers in each control volume and each edge by the following quantity

$$
\operatorname{reg}_{2}(\mathfrak{M})=\max \left(\max _{\substack{\mathcal{K} \in \mathfrak{M} \\ \sigma \in \mathcal{E}_{\mathcal{K}}}} \frac{m_{\sigma}}{d_{\mathcal{K}, \sigma}}, \max _{\mathbf{v}=\sigma \mid \tilde{\sigma}} \frac{m_{\sigma}+m_{\tilde{\sigma}}}{d_{\sigma, \tilde{\sigma}}}\right) .
$$

Finally, for simplicity, we shall assume that the interior control volumes are triangles but the approach can easily be generalized to more general convex polygonal interior control volumes.

In order to obtain the semi-discrete finite volume scheme associated with problem 6.9 we integrate equation 6.9a on all control volumes $\mathcal{K} \in \mathfrak{M}$ and we integrate equation $6.9 \mathrm{~b}$ on all boundary control volumes $\sigma \in \partial \mathfrak{M}$. Then we use a consistent two-point flux approximation for the Laplace operator in $\Omega$ and for the Laplace-Beltrami operator on $\Gamma$. A solution of this scheme is thus a set of time-dependent unknowns

$$
\mathbf{u}(t)=\left(\left(u_{\mathcal{K}}(t)\right)_{\mathcal{K} \in \mathfrak{M}},\left(u_{\sigma}(t)\right)_{\sigma \in \partial \mathfrak{M}}\right) \in \mathbb{R}^{\mathfrak{M}} \times \mathbb{R}^{\partial \mathfrak{M}} .
$$

The scheme reads as follows: Find $t \mapsto \mathbf{u}(t) \in \mathbb{R}^{\mathfrak{M}} \times \mathbb{R}^{\partial \mathfrak{M}}$ such that,

$$
\left\{\begin{array}{r}
m_{\mathcal{K}} \partial_{t} u_{\mathcal{K}}+\sum_{\sigma=\mathcal{K} \mid \mathcal{L} \in \mathcal{E}_{\mathcal{K}}^{i n t}} m_{\sigma} \frac{u_{\mathcal{K}}-u_{\mathcal{L}}}{d_{\mathcal{K}, \mathcal{L}}}+\sum_{\sigma \in \mathcal{E}_{\mathcal{K}}^{\text {ext }}} m_{\sigma} \frac{u_{\mathcal{K}}-u_{\sigma}}{d_{\mathcal{K}, \sigma}}=0, \forall \mathcal{K} \in \mathfrak{M}, \\
\alpha m_{\sigma} \partial_{t} u_{\sigma}+\sum_{\mathbf{v}=\sigma \mid \tilde{\sigma}} \frac{u_{\sigma}-u_{\tilde{\sigma}}}{d_{\sigma, \tilde{\sigma}}}+m_{\sigma} u_{\sigma}-m_{\sigma} \frac{u_{\mathcal{K}}-u_{\sigma}}{d_{\mathcal{K}, \sigma}}=0, \forall \sigma \in \partial \mathfrak{M},
\end{array}\right.
$$

where, in the second formula, we conventionally denote by $\mathcal{K}$ the unique boundary control volume such that $\sigma \in \mathcal{E}_{\mathcal{K}}^{e x t}$.

We postpone the important discussion on the choice of the discrete initial condition $\mathbf{u}(0)=\mathbf{u}^{0}$ to Theorem 6.2 .

The discrete version of the $H_{\Gamma}^{1}$ norm is defined as follows

$$
\|\mathbf{u}\|_{1, \mathfrak{M}, \partial \mathfrak{M}}=\left(\|\mathbf{u}\|_{1, \mathfrak{M}}^{2}+\|\mathbf{u}\|_{0, \partial \mathfrak{M}}^{2}+\|\mathbf{u}\|_{1, \partial \mathfrak{M}}^{2}\right)^{\frac{1}{2}}
$$

where each term is given by

$$
\begin{gathered}
\|\mathbf{u}\|_{1, \mathfrak{M}}^{2}=\sum_{\sigma=\mathcal{K} \mid \mathcal{L} \in \mathcal{E}_{\text {int }}} m_{\sigma} d_{\mathcal{K}, \mathcal{L}}\left(\frac{u_{\mathcal{K}}-u_{\mathcal{L}}}{d_{\mathcal{K}, \mathcal{L}}}\right)^{2}+\sum_{\sigma \in \mathcal{E}_{\text {ext }}} m_{\sigma} d_{\mathcal{K}, \sigma}\left(\frac{u_{\mathcal{K}}-u_{\sigma}}{d_{\mathcal{K}, \sigma}}\right)^{2}, \\
\|\mathbf{u}\|_{0, \partial \mathfrak{M}}^{2}=\sum_{\sigma \in \partial \mathfrak{M}} m_{\sigma}\left(u_{\sigma}\right)^{2}, \quad \text { and } \quad\|\mathbf{u}\|_{1, \partial \mathfrak{M}}^{2}=\sum_{\mathbf{v}=\sigma \mid \tilde{\sigma}} d_{\sigma, \tilde{\sigma}}\left(\frac{u_{\sigma}-u_{\tilde{\sigma}}}{d_{\sigma, \tilde{\sigma}}}\right)^{2} .
\end{gathered}
$$

Note that, in the boundary term of the definition of $\|\cdot\|_{1, \mathfrak{M}}$, we use the same convention as in 6.10 ) for the notation $\mathcal{K}$.

A discrete energy estimate is obtained by multiplying the first equation in 6.10 by $\partial_{t} u_{\mathcal{K}}$, the second equation by $\partial_{t} u_{\sigma}$ and by summing the resulting equalities on $\mathfrak{M}$ and $\partial \mathfrak{M}$. We obtain

$$
\frac{d}{d t}\|\mathbf{u}(t)\|_{1, \mathfrak{M}, \partial \mathfrak{M}}^{2} \leq 0
$$


where we did not specify the form of the dissipation terms, since it is not important for our purpose.

This estimate shows that the discrete $H_{\Gamma}^{1}$ norm of the approximate solution decreases along the time and thus satisfies

$$
\sup _{t \in[0, T]}\|\mathbf{u}(t)\|_{1, \mathfrak{M}, \partial \mathfrak{M}} \leq\left\|\mathbf{u}^{0}\right\|_{1, \mathfrak{M}, \partial \mathfrak{M}} .
$$

This a priori estimate is the main tool to prove the convergence of the numerical method. However, in order to be useful, we see that the discrete initial data $\mathbf{u}^{0}$ needs to be a stable

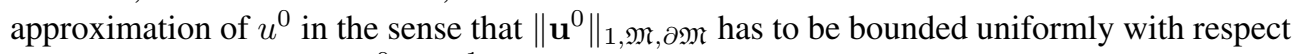
to the mesh size, for any $u^{0} \in H_{\Gamma}^{1}$.

In this framework, the inequality we proved in this paper leads to the following stability result, which was our main motivation.

THEOREM 6.2. Let $\xi_{0}>0$ and $h_{0}>0$ given by Proposition 6.1 There exists a $C>0$ such that for any finite volume mesh $\mathfrak{M}$ of $\Omega$ satisfying

$$
\operatorname{reg}_{1}(\mathfrak{M}) \leq \xi_{0}, \operatorname{reg}_{2}(\mathfrak{M}) \leq \xi_{0}, \text { and } \operatorname{size}(\mathfrak{M}) \leq h_{0},
$$

and for any $u^{0} \in H_{\Gamma}^{1}$, we have

$$
\left\|\mathbf{u}^{0}\right\|_{1, \mathfrak{M}, \partial \mathfrak{M}} \leq C\left\|u^{0}\right\|_{H_{\Gamma}^{1}}
$$

where $\mathbf{u}^{0}=\left(\left(u_{\mathcal{K}}^{0}\right)_{\mathcal{K} \in \mathfrak{M}},\left(u_{\sigma}^{0}\right)_{\sigma \in \partial \mathfrak{M}}\right)$ is defined by

$$
\left\{\begin{array}{l}
u_{\mathcal{K}}^{0}=\frac{1}{m_{\mathcal{K}}} \int_{\mathcal{K}} u^{0}(x) d x, \quad \forall \mathcal{K} \in \mathfrak{M}, \\
u_{\sigma}^{0}=\frac{1}{m_{\sigma}} \int_{\sigma} u_{\mid \Gamma}^{0} d x, \quad \forall \sigma \in \partial \mathfrak{M} .
\end{array}\right.
$$

Notice first that, in order to take advantage of the assumed regularity of the trace of $u^{0}$ on $\Gamma$ in the estimate of the tangential gradient term $\left\|\mathbf{u}^{0}\right\|_{1, \partial \mathfrak{M}}$, we absolutely need to define the boundary terms $\mathbf{u}_{\sigma}^{0}$ by using only the values of the trace of $u^{0}$ on $\Gamma$ and not, for instance, the values of $u^{0}$ on the chords associated with each boundary control volume $\sigma$.

Proof.

- The estimate of the $L^{2}$ term $\left\|\mathbf{u}^{0}\right\|_{0, \partial \mathfrak{M}}$ is a straightforward consequence of Jensen's inequality.

- For any two neighboring boundary control volumes $\sigma$ and $\tilde{\sigma}$, one can easily prove by using a Taylor formula on the manifold $\Gamma$, that

$$
\left|\frac{1}{m_{\sigma}} \int_{\sigma} u^{0}-\frac{1}{m_{\tilde{\sigma}}} \int_{\tilde{\sigma}} u^{0}\right|^{2} \leq\left(m_{\sigma}+m_{\tilde{\sigma}}\right) \int_{\sigma \cup \tilde{\sigma}}\left|\nabla_{\Gamma} u^{0}\right|^{2} .
$$

It follows that

$$
\begin{aligned}
\left\|\mathbf{u}^{0}\right\|_{1, \partial \mathfrak{M}}^{2} & =\sum_{\mathbf{v}=\sigma \mid \tilde{\sigma}} \frac{1}{d_{\sigma, \tilde{\sigma}}}\left(\frac{1}{m_{\sigma}} \int_{\sigma} u^{0}-\frac{1}{m_{\tilde{\sigma}}} \int_{\tilde{\sigma}} u^{0}\right)^{2} \\
& \leq \sum_{\mathbf{v}=\sigma \mid \tilde{\sigma}} \frac{m_{\sigma}+m_{\tilde{\sigma}}}{d_{\sigma, \tilde{\sigma}}} \int_{\sigma \cup \tilde{\sigma}}\left|\nabla_{\Gamma} u^{0}\right|^{2} \\
& \leq 2 \operatorname{reg}_{2}(\mathfrak{M}) \int_{\Gamma}\left|\nabla_{\Gamma} u^{0}\right|^{2} .
\end{aligned}
$$


- It remains to estimate the term $\left\|\mathbf{u}^{0}\right\|_{1, \mathfrak{M}}^{2}$. To this end, we first estimate the term corresponding to the interior edges as follows

$$
\begin{aligned}
\sum_{\sigma=\mathcal{K} \mid \mathcal{L} \in \mathcal{E}_{i n t}} m_{\sigma} d_{\mathcal{K}, \mathcal{L}}\left(\frac{u_{\mathcal{K}}^{0}-u_{\mathcal{L}}^{0}}{d_{\mathcal{K}, \mathcal{L}}}\right)^{2} & =\sum_{\sigma=\mathcal{K} \mid \mathcal{L} \in \mathcal{E}_{\text {int }}} \frac{m_{\sigma}}{d_{\mathcal{K}, \mathcal{L}}}\left(u_{\mathcal{K}}^{0}-u_{\mathcal{L}}^{0}\right)^{2} \\
& \leq 2 \sum_{\sigma=\mathcal{K} \mid \mathcal{L} \in \mathcal{E}_{i n t}} \frac{m_{\sigma}}{d_{\mathcal{K}, \mathcal{L}}}\left[\left(u_{\mathcal{K}}^{0}-u_{\sigma}^{0}\right)^{2}+\left(u_{\sigma}^{0}-u_{\mathcal{L}}^{0}\right)^{2}\right],
\end{aligned}
$$

where we have introduced the mean-values on the edges $u_{\sigma}^{0}$ as in 6.11 but for interior edges now.

Gathering this computation with the other term in $\left\|\mathbf{u}^{0}\right\|_{1, \mathfrak{M}}^{2}$, we obtain

$$
\left\|\mathbf{u}^{0}\right\|_{1, \mathfrak{M}}^{2} \leq 2 \sum_{\mathcal{K} \in \mathfrak{M}} \sum_{\sigma \in \mathcal{E}_{\mathcal{K}}} \frac{m_{\sigma}}{d_{\mathcal{K}, \sigma}}\left(u_{\mathcal{K}}^{0}-u_{\sigma}^{0}\right)^{2}
$$

We can now use Theorem 2.1 and Proposition 6.1, to obtain

$$
\begin{aligned}
\left\|\mathbf{u}^{0}\right\|_{1, \mathfrak{M}}^{2} & \leq C_{\xi_{0}} \sum_{\mathcal{K} \in \mathfrak{M}} \sum_{\sigma \in \mathcal{E}_{\mathcal{K}}} \frac{m_{\sigma}}{d_{\mathcal{K}, \sigma}}\left(m_{\sigma}+\operatorname{diam}(\mathcal{K})\right)^{2} \frac{1}{m_{\mathcal{K}}} \int_{\mathcal{K}}\left|\nabla u^{0}\right|^{2} \\
& \leq C_{\xi_{0}} \operatorname{reg}_{2}(\mathfrak{M})\left(1+\operatorname{reg}_{2}(\mathfrak{M})\right)^{2} \sum_{\mathcal{K} \in \mathfrak{M}} \sum_{\sigma \in \mathcal{E}_{\mathcal{K}}} \operatorname{diam}(\mathcal{K})^{2} \frac{1}{m_{\mathcal{K}}} \int_{\mathcal{K}}\left|\nabla u^{0}\right|^{2} \\
& \leq 3 C_{\xi_{0}} \operatorname{reg}_{2}(\mathfrak{M})\left(1+\operatorname{reg}_{2}(\mathfrak{M})^{2}\right) \operatorname{reg}_{1}(\mathfrak{M}) \int_{\Omega}\left|\nabla u^{0}\right|^{2},
\end{aligned}
$$

and the claim is proved. Notice that the assumptions of Theorem 2.1 are satisfied with a uniform ratio $\nu / \mu$ thanks to Proposition 6.1. and to the fact that for interior control volumes $\mathcal{K}$, which are real triangles, the ratio $\nu / \mu$ is equal to 1 (see Remark 2.1.

\section{Appendix A. An intermediate result.}

LEMMA A.1. There exists a $C^{1}$ diffeomorphism $\psi: \overline{Q^{n-1}} \rightarrow \overline{Q^{n-1}}$ such that, setting $\widetilde{\gamma}=\gamma \circ \psi$, for every $\theta \in \overline{Q^{n-1}}$,

$$
\left|\partial_{1} \widetilde{\gamma} \wedge \cdots \wedge \partial_{n-1} \widetilde{\gamma}\right|(\theta)=m_{\sigma} .
$$

Proof. This follows from the proof of [8, Lemma 2], see also [3, Theorem 7, Proposition A.2]. However, in the former reference, all the data are assumed to be smooth. In the latter (which gives a result on more general domains than a cube), the map $\psi$ is merely $C^{1}$ on $Q^{n-1}$ instead of $\overline{Q^{n-1}}$. For the convenience of the reader, we detail the proof.

Let $f=\frac{1}{m_{\sigma}}\left|\partial_{1} \gamma \wedge \cdots \wedge \partial_{n-1} \gamma\right|$. Then $\int_{Q^{n-1}} f=1$.

and

For every $k=1, \ldots, n-1$, there exists a $C^{1}$ map $f_{k}: \overline{Q^{k}} \rightarrow \mathbb{R}$ such that $f=f_{1} \ldots f_{n-1}$

$$
\int_{0}^{1} f_{k}\left(x_{1}, \ldots, x_{k-1}, t\right) d t=1 \quad, \quad\left(x_{1}, \ldots, x_{k-1}\right) \in \overline{Q^{k-1}}
$$

Indeed, let

$$
f_{1}\left(x_{1}\right)=\int_{Q^{n-2}} f\left(x_{1}, t_{2}, \ldots, t_{n-1}\right) d t_{2} \ldots d t_{n-1} \quad, \quad x_{1} \in[0,1]
$$


and then define by induction on $1 \leq k \leq n-1$, the map $f_{k}$ by

$$
f_{1}\left(x_{1}\right) \ldots f_{k}\left(x_{1}, \ldots, x_{k}\right)=\int_{Q^{n-1-k}} f\left(x_{1}, \ldots, x_{k}, t_{k+1}, \ldots, t_{n-1}\right) d t_{k+1} \ldots d t_{n-1} .
$$

(When $k=n-1$, the right-hand side is simply $f\left(x_{1}, \ldots, x_{n-1}\right)$.) One easily checks that A.1 is satisfied. We now define the map $\rho=\left(\rho_{1}, \ldots, \rho_{n-1}\right)$ by

$$
\rho_{i}\left(x_{1}, \ldots, x_{i}\right)=\int_{0}^{x_{i}} f_{i}\left(x_{1}, \ldots, x_{i-1}, t\right) d t \quad, \quad 1 \leq i \leq n-1 .
$$

Then $x_{i} \mapsto \rho_{i}\left(x_{1}, \ldots, x_{i}\right)$ maps diffeomorphically $[0,1]$ onto $[0,1]$. Moreover,

$$
\operatorname{Jac} \rho=\prod_{i=1}^{n-1} \frac{\partial \rho_{i}}{\partial x_{i}}=\prod_{i=1}^{n-1} f_{i}=f .
$$

We then define $\psi=\rho^{-1}$. Then

$$
1=\operatorname{Jac}(\rho \circ \psi)=((\mathbf{J a c} \rho) \circ \psi) \mathbf{J a c} \psi=(f \circ \psi) \mathbf{J a c} \psi .
$$

Since

$$
\partial_{1} \widetilde{\gamma} \wedge \cdots \wedge \partial_{n-1} \widetilde{\gamma}=\left(\left(\partial_{1} \gamma \wedge \cdots \wedge \partial_{n-1} \gamma\right) \circ \psi\right) \operatorname{Jac} \psi,
$$

this completes the proof of the lemma.

Acknowledgements. The authors want to warmly thank the anonymous referees for their very careful reading of the paper and their useful comments.

\section{REFERENCES}

[1] B. Andreianov, F. Boyer, and F. Hubert. Discrete duality finite volume schemes for Leray-Lions type elliptic problems on general 2D-meshes. Num. Meth. for PDEs, 23(1):145-195, 2007.

[2] Susanne C. Brenner and L. Ridgway Scott. The mathematical theory of finite element methods, volume 15 of Texts in Applied Mathematics. Springer, New York, third edition, 2008.

[3] B. Dacorogna and J. Moser. On a partial differential equation involving the Jacobian determinant. Ann. Inst. H. Poincaré Anal. Non Linéaire, 7(1):1-26, 1990.

[4] J. Droniou. Error estimates for the convergence of a finite volume discretization of convection-diffusion equations. J. Numer. Math., 11(1):1-32, 2003.

[5] J. Droniou and R. Eymard. A mixed finite volume scheme for anisotropic diffusion problems on any grid. Numer. Math., 105(1):35-71, 2006.

[6] J. Droniou and R. Eymard. Study of the mixed finite volume method for Stokes and Navier-Stokes equations. Num. Meth. for PDEs, 25(1):137-171, 2009.

[7] R. Eymard, T. Gallouët, and R. Herbin. Finite volume methods. In Ph Ciarlet and J.L. Lions, editors, Handbook of numerical analysis, Vol. VII, Handb. Numer. Anal., VII, pages 715-1022. North-Holland, Amsterdam, 2000.

[8] J. Moser. On the volume elements on a manifold. Trans. Amer. Math. Soc., 120:286-294, 1965.

[9] F. Nabet. Convergence of a finite-volume scheme for the Cahn-Hilliard equation with dynamic boundary conditions. IMA J. Num. Anal., 2015. to appear, http://hal.archives-ouvertes.fr/ hal-01096996

[10] J.L. Vázquez and E. Vitillaro. Heat equation with dynamical boundary conditions of reactive-diffusive type. Journal of Differential Equations, 250(4):2143 - 2161, 2011. 